\title{
Morse Theory for S-balanced Configurations in the Newtonian $n$-body Problem
}

\author{
Luca Asselle $^{1}$ (D) Alessandro Portaluri $^{2}$ (D)
}

Received: 14 November 2020 / Revised: 15 April 2021 / Accepted: 31 May 2021 /

Published online: 22 July 2021

(c) The Author(s) 2021

\begin{abstract}
For the Newtonian (gravitational) $n$-body problem in the Euclidean $d$-dimensional space, the simplest possible solutions are provided by those rigid motions (homographic solutions) in which each body moves along a Keplerian orbit and the configuration of the $n$-body is a (constant up to rotations and scalings) central configuration. For $d \leq 3$, the only possible homographic motions are those given by central configurations. For $d \geq 4$ instead, new possibilities arise due to the higher complexity of the orthogonal group $\mathrm{O}(d)$, as observed by Albouy and Chenciner (Invent Math 131(1):151-184, 1998). For instance, in $\mathbb{R}^{4}$ it is possible to rotate in two mutually orthogonal planes with different angular velocities. This produces a new balance between gravitational forces and centrifugal forces providing new periodic and quasi-periodic motions. So, for $d \geq 4$ there is a wider class of $S$-balanced configurations (containing the central ones) providing simple solutions of the $n$-body problem, which can be characterized as well through critical point theory. In this paper, we first provide a lower bound on the number of balanced (non-central) configurations in $\mathbb{R}^{d}$, for arbitrary $d \geq$ 4 , and establish a version of the $45^{\circ}$-theorem for balanced configurations, thus answering some of the questions raised in Moeckel (Central configurations, 2014). Also, a careful study of the asymptotics of the coefficients of the Poincare polynomial of the collision free configuration sphere will enable us to derive some rather unexpected qualitative consequences on the count of $S$-balanced configurations. In the last part of the paper, we focus on the case $d=4$ and provide a lower bound on the number of periodic and quasi-periodic motions of the gravitational $n$-body problem which improves a previous celebrated result of McCord (Ergodic Theory Dyn Syst 16:1059-1070, 1996).
\end{abstract}

Keywords $n$-body problem · Balanced configurations · Central configurations · $45^{\circ}$-Theorem

$\triangle$ Luca Asselle

luca.asselle@math.uni-giessen.de

Alessandro Portaluri

alessandro.portaluri@unito.it

1 Justus Liebig Universität Gießen, Arndtstrasse 2, 35392 Gießen, Germany

2 Università degli Studi di Torino, Largo Paolo Braccini, 2, 10095 Grugliasco, Torino, Italy 


\section{Contents}

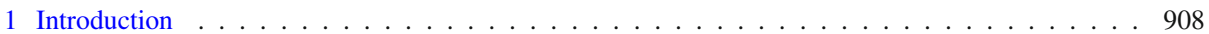

2 S-balanced Configurations: Definition and Basic Properties . . . . . . . . . . . . . . . . . . . 912

$2.1 \mathrm{CSBC}$ and Assumption $(\mathrm{H} 1) \ldots \ldots \ldots \ldots \ldots \ldots \ldots$

2.2 CSBC and Corresponding CCC: Spectra and Inertia Indices . . . . . . . . . . . . . . . . 918

3 Estimates on the Coefficients of the Poincaré Polynomial . . . . . . . . . . . . . . . . . . . . 922

4 Lower Bounds on the Number of SBC . . . . . . . . . . . . . . . . . . . . . . . . . . . 926

5 The $45^{\circ}$ Theorem for Balanced Configurations . . . . . . . . . . . . . . . . . . . . . . . . . . . 932

6 A Lower Bound on the Number of SBC in $\mathbb{R}^{4}$ á la McCord . . . . . . . . . . . . . . . . . . . . . 937

6.1 Homology of Some Intermediate Manifolds . . . . . . . . . . . . . . . . . . . . . . . 938

6.2 The Homology of $\overline{\mathcal{S}} \ldots \ldots \ldots \ldots$. . . . . . . . . . . . . . . . . . . . 940

Appendix: A Proof of Lemma $3.8 \ldots \ldots \ldots$. . . . . . . . . . . . . . . . . . . . . . . . 944

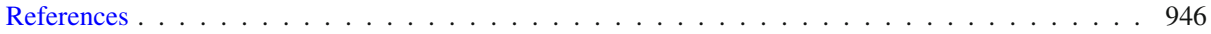

\section{Introduction}

The Newtonian $n$-body problem concerns the motion of $n$ point particles with masses $m_{j} \in$ $\mathbb{R}^{+}$and position $q_{j} \in \mathbb{R}^{d}$, where $j=1, \ldots, n$ and $d \geq 2$, interacting each other according to Newton's law of inverses squares. The equations of motion read

$$
m_{j} \ddot{q}_{j}=\frac{\partial U}{\partial q_{j}}, \quad \text { where } \quad U\left(q_{1}, \ldots, q_{n}\right):=\sum_{i<j} \frac{m_{i} m_{j}}{\left|q_{i}-q_{j}\right|} .
$$

As the center of mass has an inertial motion, there is no loss in generalities in assuming that the center of mass lies at the origin. The CONFIGURATION SPACE WITH CENTER OF MASS AT THE ORIGIN is defined as

$$
\mathbb{X}:=\left\{\left(q_{1}, \ldots, q_{n}\right) \in \mathbb{R}^{d n} \mid \sum_{i=1}^{n} m_{i} q_{i}=0\right\} .
$$

The potential $U$ is not defined when (at least) two particles collide, meaning that they have the same position. Therefore, we define the space of COLLISION FREE CONFIGURATIONS as

$$
\widehat{\mathbb{X}}:=\left\{q=\left(q_{1}, \ldots, q_{n}\right) \in \mathbb{X} \mid q_{i} \neq q_{j} \text { for } i \neq j\right\}=\mathbb{X} \backslash \Delta,
$$

where

$$
\Delta:=\left\{q=\left(q_{1}, \ldots, q_{n}\right) \in \mathbb{R}^{d n} \mid q_{i}=q_{j} \text { for } i \neq j\right\}
$$

is the COLLISION SET. Let $M$ be the $(n d \times n d)$-diagonal MASS MATRIX defined by

$$
M:=\operatorname{diag}(\underbrace{m_{1}, \ldots, m_{1}}_{d \text {-times }}, \ldots, \underbrace{m_{n}, \ldots, m_{n}}_{d \text {-times }})
$$

and let

$$
\langle\cdot, \cdot\rangle_{M}:=\langle M \cdot, \cdot\rangle \quad \text { and } \quad|\cdot|_{M}:=\langle M \cdot, \cdot\rangle^{1 / 2}
$$

be respectively the MASS SCALAR PRODUCT and the MASS NORM, where $\langle\cdot, \cdot\rangle$ denotes the Euclidean product in $\mathbb{R}^{n d}$. In this notation, the equations of motion can be written as

$$
\ddot{q}=M^{-1} \nabla U(q) .
$$


Among all possible configurations of the system, a crucial role in penetrating the intricate dynamics of this problem, is played by the so-called CENTRAL CONFIGURATIONS (CC in shorthand notation), namely configurations in which $M^{-1} \nabla U(q)$ is parallel to $q$ :

$$
M^{-1} \nabla U(q)+\lambda q=0
$$

In other words, the acceleration vector of each particle is pointing towards the origin with magnitude proportional to the distance to the origin. As a straightforward consequence of the homogeneity of the potential we obtain that the proportionality constant $\lambda$ is actually equal to $-U(q) /|q|_{M}^{2}$.

Equation (1.3) is a nonlinear algebraic equation which turns out to be extremely hard to solve. Despite substantial progresses (starting from the work of - among others-Smale, Conley, Albouy, Chenciner, McCord, Moeckel, Pacella) have been made in the last decades, many basic questions about $\mathrm{CC}$ still remain unsolved. Nevertheless, there are several reasons why CC are of interest in the $n$-body problem and more generally in Celestial Mechanics:

- Every CC defines a HOMOTHETIC SOLUTION of (1.2), namely a solution which preserves its shape for all time while receding from or collapsing into the center of mass.

- Planar CC give rise to a family of periodic motions of (1.2), the so-called RELATIVE EQUILIBRIA, in which each of the bodies moves on a circular Kepler orbit. In other words, the configuration rigidly rotates at a constant angular speed about the center of mass. These motions are true equilibrium solutions in a uniformly rotating coordinate system and correspond to elliptical orbits of the Kepler problem of eccentricity $e=0$. It is worth observing that relative equilibria are the simplest periodic solutions of the $n$-body problem having an explicit formula.

- Planar CC more generally give rise to a family of HOMOGRAPHIC SOLUTIONS of (1.2) in which each particle traverses an elliptical orbit with eccentricity $e \in(0,1)$. In this case, both the radius $r(t)$ of the orbit and the angular speed $\dot{\vartheta}(t)$ are time-dependent solutions of the Kepler problem (in polar coordinates). Moreover, the configuration remains similar to the initial configuration throughout the motion, varying only in size.

- CC control the qualitative behavior of an important class of colliding solutions (and parabolic motions) of the $n$-body problem. More precisely, if an orbit approaches or recedes from total collapse, it does so approximating homothetic orbits. So, orbits passing arbitrarily close to a total collision do it by first approaching one homothetic motion and then receding from the collision following a (possibly different) homothetic motion.

In other words, for the $n$-body problem in $\mathbb{R}^{d}, d \leq 3$, any CC generates a homothetic ejection or collapse. Morevorer, any planar CC generates planar Keplerian homographic motions, whereas spatial non-planar CC can only produce homothetic motions. Configurations which are not central cannot produce homographic motions at all. If we instead allow dimensions $d \geq 4$, then — as observed by Albouy and Chenciner in [1] (cfr. also [9]) — there is a wider class of the so-called " $S$-balanced configurations" which produces relative equilibria of the $n$-body problem. These new phenomena are due to the higher complexity of the group of rotations in higher dimensions, which allows, for example, to rotate in two mutually orthogonal planes with different angular velocities. This leads to new ways of balancing the gravitational forces with centrifugal forces in order to get new relative equilibria of the $n$-body problem. We shall notice that, in contrast with the case $d=2$, such relative equilibria need not be periodic in time. Indeed, if the angular velocities are rationally independent, then the resulting motions will be only quasi-periodic. 
In order to formally define such a class of configurations, we consider positive real numbers $s_{1} \geq \ldots \geq s_{d}>0$ (possibly not all different) and set

$$
\widehat{S}:=\operatorname{diag}(\underbrace{S, \ldots, S}_{n \text {-times }}) \in \mathbb{R}^{n d \times n d},
$$

where $S=\operatorname{diag}\left(s_{1}, \ldots, s_{d}\right)$. An $S$ - BALANCED CONFIGURATION, (SBC in shorthand notation), is an arrangement of the masses whose associated configuration vector $q \in \widehat{\mathbb{X}}$ satisfies

$$
\nabla U(q)+\lambda \widehat{S} M q=0
$$

for some real (positive) constant $\lambda$. We observe that for $n=3$ a planar non-equilateral and non-collinear isosceles triangle is a SBC (but not a CC) as soon as the two symmetric masses are equal. Also, the class of SBC strictly includes CC (corresponding, in fact, to $S=I$ ), and Eq. (1.4) is in general only invariant under the (diagonal) action of a subgroup of $\mathrm{SO}(d)$, which is proper as soon as $S \neq I$. In the extremal case in which the (diagonal) entries of $S$ are pairwise distinct, the symmetry group of Eq. (1.4) is trivial.

Remark 1.1 The usual definition of $S$-balanced configurations (cfr. e.g. [9]) requires $S$ to be a symmetric positive definite matrix. However, since the problem is invariant by unitary transformation, there is no loss of generality in assuming that $S$ be in diagonal form, i.e. that the spectral basis coincide with the canonical basis of $\mathbb{R}^{d}$. Also, one requires $S$ to be minus the square of a skew-symmetric matrix (e.g. a complex structure), and hence, in particular, that all eigenvalues of $S$ have even multiplicity. As we shall see later, it will be very convenient to allow $S$ to have eigenvalues of odd multiplicity (in particular simple).

It is worth pointing out that, in even dimensions, if $S=-A^{2}$ where $A$ is an antisymmetric matrix, every SBC gives rise to a uniformly rotating relative equilibrium solution of Eq. (1.2). To get more general homographic solutions it is still necessary to have a $\mathrm{CC}$ in which the bodies run on planar Keplerian ellipses. However, ellipses corresponding to different bodies may lie in different planes.

In order to find solutions of (1.4), we will exploit the fact that SBC (as well as CC) admit a variational characterization as critical points of the restriction of $U$ to the COLLISION FREE CONFIGURATION SPHERE

$$
\widehat{\mathbb{S}}:=\mathbb{S} \backslash \Delta, \quad \text { where } \quad \mathbb{S}:=\left\{q \in \mathbb{X} \mid I_{S}(q)=1\right\}
$$

is the CONFIGURATION SPHERE and $I_{S}(q)$ if the S- WEIGHTED MOMENT OF INERTIA, namely the norm squared associated to the scalar product induced by $\widehat{S} M$. The proof of Shub's lemma carries over word by word to this more general situation, thus showing that SBC cannot accumulate on the collision set $\Delta$. In particular, we can find a neighborhood of $\Delta$ in $\mathbb{S}$ which contains no SBC. This opens up the possibility of using Morse theoretical methods to obtain lower bounds on the number of solutions to (1.4). However, in doing this, one has to keep in mind that:

- Equation (1.4) has a non-trivial symmetry group whenever at least two entries of the matrix $S$ are equal, and hence critical points are not isolated unless all entries of $S$ are distinct. Also, such an action is not free (actually, non even locally free) as soon as $d \geq 3$. Therefore, the quotient space is in general not even an orbifold but rather an Alexandrov space, and hence a delicate stratified Morse theory is needed when working on the quotient space. Methods such as Morse-Bott theory, which allow to deal with functions having 
(Morse-Bott) non-degenerate critical manifolds, do not take into account the symmetries of the problem and hence necessarily lead to weaker results.

- Even if one is able to give lower bounds on the number of SBC, for instance via equivariant Morse theory or Morse-Bott theory, one has additionally to show that the SBC that one finds are actually not $\mathrm{CC}$. This is the same problem that one has to face when trying to exclude that spatial CC are actually not planar: despite the lower bounds provided by Pacella [11], it is so far not known whether there is more than one spatial CC which is not planar.

- All previously mentioned methods are essentially based on equivariant homology and intersection homology theories, respectively, which are computationally inaccessible in full generality.

We will circumvent all these difficulties at once by restricting our attention to a subspace of $\mathbb{R}^{d}$ in which the eigenvalues of $S$ are pairwise distinct (in what follows this will be called the "reduction to Assumption (H1)" argument). Assumption (H1) will kill all symmetries of Eq. (1.4), thus allowing us to apply standard Morse theory arguments. Also, the fact that all entries of $S$ are distinct implies that solutions of (1.4) which are not collinear cannot be CC. More will be said about Assumption (H1) in Sect. 2. Therefore, applying classical Morse theory to $\widehat{U}:=\left.U\right|_{\widehat{S}}$ under Assumption (H1), we derive lower bounds on the number of SBC assuming non-degeneracy. These lower bounds will depend on suitable spectral gap conditions involving the eigenvalues of the matrix $S$ (cfr. Theorems 4.2, 4.5 for the precise statements). A first key step in this direction is provided by a careful investigation of collinear SBC (in shorthand notation CSBC) and their inertia indices (cfr. Lemma 2.13 for further details). The Poincaré polynomial of $\mathbb{S}$ is the same as in the case of $\mathrm{CC}$, however establishing precise growth estimates on its coefficients (cfr. Proposition 3.2) we are able to derive nontrivial and rather unexpected qualitative consequences on the count of SBC: roughly speaking, CSBC corresponding to smaller eigenvalues of $S$ will in many cases contribute more than CSBC corresponding to the largest eigenvalue of $S$ (this should be compared with $[9, \mathrm{P}$. 151]). We stress the fact that all lower bounds obtained via the reduction to (H1) argument provide genuine SBC.

In the last section we prove a sharper lower bound on the number of non-degenerate SBC in dimension $d=4$ for $S=\operatorname{diag}(s, s, 1,1)$. Even if the proof is again based on Morse theory, the argument is completely different than the one used in the proof of Theorem 4.2. After restricting our attention to the $\{0\} \times \mathbb{R}^{3} \subset \mathbb{R}^{4}$, we quotient out $\widehat{\mathbb{S}}$ by the $\mathrm{SO}(2)$-action given by rotations in the $\{0\} \times \mathbb{R}^{2}$-plane after removing the manifold $\mathbb{Y}$ of collinear configurations contained in $\mathbb{R} \times\{0\}$. This is done by first establishing a so-called $45^{\circ}$ THEOREM FOR SBC (cfr. Theorem 5.2). Therefore, the quotient

$$
\overline{\mathcal{S}}=(\widehat{\mathbb{S}} \backslash \mathbb{Y}) / \mathrm{SO}(2)
$$

is a manifold. We then compute in Theorem 6.4 the homology of $\overline{\mathcal{S}}$ by using Alexander duality and the Gysin long exact sequence, and finally obtain the desired lower bound by explicitly computing the sum of the Betti numbers of $\overline{\mathcal{S}}$. This has an important consequence on the lower bound of periodic orbits of the gravitational $n$-body problem. In fact, assuming non-degeneracy, we have at least

$$
n !\left(h(n)+\frac{1}{2}+\frac{1}{n}\right), \quad h(n):=\sum_{j=3}^{n} \frac{1}{j},
$$


relative equilibria in $\mathbb{R}^{4}$ for fixed $s>1$. Such a number is roughly twice as large as the lower bound proved by McCord in [7]: among all of these relative equilibria, at least

$$
\frac{n !}{2}(h(n)+1)
$$

are produced by CC, namely $n ! h(n) / 2$ corresponding to planar non-collinear CC (McCord's estimate) and $n ! / 2$ corresponding to collinear CC (CCC in shorthand notation). By subtracting the latter integer to the former one, we get at least

$$
n !\left(\frac{h(n)}{2}+\frac{1}{n}\right)
$$

relative equilibria which do not come from McCord's estimate, but could anyway come from CC (unlike in Theorem 4.2, this cannot be excluded a priori). Finally, we observe that the relative equilibria coming from genuine $\mathrm{SBC}$ are periodic in time for $s \in \mathbb{Q}$, and quasiperiodic otherwise.

We finish this introduction by summarizing, for the reader's sake, some of the notation that we shall use henceforth throughout the paper.

- $M$ is the $n d$-dimensional mass matrix; $I_{d}$ is the $d$-dimensional identity matrix; $S$ is a $d$-dimensional diagonal matrix, $\widehat{S}:=\operatorname{diag}(S, \ldots, S)$.

- $\langle\cdot, \cdot\rangle_{M},|\cdot|_{M}$ denote respectively the mass scalar product and the mass norm.

- $I(q), I_{S}(q)$ denote the moment of inertia and the $S$-weighted moment of inertia (w.r.t. origin).

- $\mathbb{X}$ is the configuration space with center of mass at the origin; $\Delta$ is the collision set and $\widehat{\mathbb{X}}:=X \backslash \Delta$ is the collision free configuration space; $\mathbb{S}$ is the configuration sphere and $\widehat{\mathbb{S}}:=\mathbb{S} \backslash \Delta$ is the collision free configuration sphere.

- $\widehat{U}:=\left.U\right|_{\widehat{\mathbb{S}}}$ is the restriction of $U$ to $\widehat{\mathbb{S}}$.

- CC, SBC, CCC, CSBC denote respectively the set of central configurations, $S$-balanced configurations, collinear CC and finally collinear SBC.

- $\iota^{-}(q), \iota^{+}(q), \iota^{0}(q)$ denote respectively the Morse index, the Morse co-index, and the nullity of the SBC $q$ as a critical point of $\widehat{U}$.

\section{S-balanced Configurations: Definition and Basic Properties}

This section is devoted to recall the definition of $S$-balanced configurations and to collect some of their properties. Our basic reference, for this section, is [9].

For $n \geq 2$, let $m_{1}, \ldots, m_{n}$ be positive real numbers (which can be thought of as the masses of the $n$ points) and let $M$ be the diagonal (block) ( $n d \times n d$ )-matrix defined as

$$
M:=\left[M_{i j}\right]_{i, j=1}^{n}, \quad M_{i j}:=m_{j} \delta_{i j} I_{d},
$$

where $I_{d}$ denotes the $d$-dimensional identity matrix, $d \geq 1$. Denoting by $\langle\cdot, \cdot\rangle$ the Euclidean product in $\mathbb{R}^{\text {nd }}$, we denote by

$$
\langle\cdot, \cdot\rangle_{M}:=\langle M \cdot, \cdot\rangle \quad \text { and } \quad|\cdot|_{M}:=\langle M \cdot, \cdot\rangle^{1 / 2}
$$

respectively the MASS SCALAR PRODUCT and the MASS NORM. As the center of mass has an inertial motion, it is not restrictive to prescribe its position at the origin. Therefore, we define 
the CONFIGURATION SPACE WITH CENTER OF MASS AT THE ORIGIN as

$$
\mathbb{X}:=\left\{\left(q_{1}, \ldots, q_{n}\right) \in \mathbb{R}^{d n} \mid \sum_{i=1}^{n} m_{i} q_{i}=0\right\} .
$$

It is readily seen that $\mathbb{X}$ is an $N$-dimensional (real) vector space where $N:=d(n-1)$. We define the space of COLLISION FREE CONFIGURATIONS as

$$
\widehat{\mathbb{X}}:=\left\{q=\left(q_{1}, \ldots, q_{n}\right) \in \mathbb{X} \mid q_{i} \neq q_{j} \text { for } i \neq j\right\}=\mathbb{X} \backslash \Delta,
$$

where

$$
\Delta:=\left\{q=\left(q_{1}, \ldots, q_{n}\right) \in \mathbb{R}^{d n} \mid q_{i}=q_{j} \text { for } i \neq j\right\}
$$

denotes the COLLISION SET. The NeWTONIAN POTENTIAL $U: \widehat{\mathbb{X}} \rightarrow \mathbb{R}$ at the CONFIGURATION VECTOR $q$ is given by

$$
U(q):=\sum_{i<j} \frac{m_{i} m_{j}}{\left|q_{i}-q_{j}\right|} .
$$

and so, Newton's equations of motion read as follows

$$
M \ddot{q}=\nabla U(q)=\sum_{i \neq j} \frac{m_{i} m_{j}}{\left|q_{i}-q_{j}\right|^{3}} \cdot\left(q_{i}-q_{j}\right),
$$

where $\nabla U$ denotes the $n d$-dimensional gradient. Given positive real numbers $s_{1} \geq \ldots \geq$ $s_{d}>0$ (possibly not all different), we let $S$ be the diagonal $(d \times d)$-matrix defined by $S=\operatorname{diag}\left(s_{1}, \ldots, s_{d}\right)$, and

$$
\widehat{S}:=\operatorname{diag}(\underbrace{S, \ldots, S}_{n \text {-times }}) \in \mathbb{R}^{n d \times n d} .
$$

Definition 2.1 An S-balanced configuration, (SBC in shorthand notation), is an arrangement of the masses whose associated configuration vector $q \in \widehat{\mathbb{X}}$ satisfies

$$
\nabla U(q)+\lambda \widehat{S} M q=0
$$

for some real (positive) constant $\lambda$.

Remark 2.2 In [9] the matrix $S$ is assumed to be symmetric and positive definite. Nevertheless, we are not losing generality by assuming here that $S$ is in diagonal form. Indeed, a symmetric matrix can always be diagonalized by choosing a spectral basis. In other words, here we are simply assuming that the spectral basis coincides with the standard basis of $\mathbb{R}^{d}$. This is possible since the formulation of the problem doesn't depend upon this choice. Also, one usually assumes that $S$ be minus the square of a skew-symmetric matrix, hence in particular that each eigenvalue of $S$ have even multiplicity. As we shall see later, it will be very convenient to allow $S$ to have eigenvalues of odd multiplicity, in particular simple eigenvalues.

The two extreme cases correspond to $s_{1}=\ldots=s_{d}=1$, respectively to $s_{i} \neq s_{j}$ for all $i \neq j$. In the former case we get the well-known notion of CENTRAL CONFIGURATION (CC for short) for which a rich literature is nowadays available, whereas the latter case will be the main object of interest of the present paper. 
Definition 2.3 The MOMENT OF INERTIA (W.R.T. $O$ ) for the configuration vector $q$ is defined by

$$
I(q):=|q|_{M}^{2} .
$$

Analogously, we term $S$ - WEIGHTED MOMENT OF INERTIA (W.R.T. $O$ ) the positive number

$$
I_{S}(q):=\langle\widehat{S} M q, q\rangle=|q|_{S}^{2}
$$

where $|\cdot|_{S}^{2}$ denotes the norm squared induced by the scalar product $\langle\cdot, \cdot\rangle_{S}:=\langle\widehat{S} M \cdot, \cdot\rangle$.

Remark 2.4 Taking the scalar product of Eq. (2.1) with $q$ and using Euler's theorem for positively homogeneous functions, we get that the (positive) constant $\lambda$ appearing in Eq. (2.1) is given by

$$
\lambda:=\frac{U(q)}{I_{S}(q)} .
$$

In particular, for any $\operatorname{SBC} q$, we get a continuous family of SBC by scaling.

Definition 2.5 Under the previous notation, we define the CONFIGURATION SPHERE and the COLLISION FREE CONFIGURATION SPHERE as follows

$$
\mathbb{S}:=\left\{q \in \mathbb{X} \mid I_{S}(q)=1\right\} \quad \text { and } \quad \widehat{\mathbb{S}}:=\mathbb{S} \backslash \Delta
$$

We refer to its elements as (NORMALIZED) CONFIGURATION VECTORS.

It is immediate to check that $\mathbb{S}$ is a smooth compact manifold diffeomorphic to a $(N-1)$ dimensional sphere. As a direct consequence of the scaling property of Eq. (2.1), with any $\mathrm{SBC}$ we can associate a unique normalized SBC. Hereafter, we will refer to normalized SBC simply as SBC.

The next result can be obtained by a straightforward modification of the proof of the variational characterization of $\mathrm{CC}$ and provides a variational characterization of SBC as critical points of the restriction of $U$ to the collision free configuration sphere.

Lemma 2.6 A (normalized) configuration vector $q$ is an SBC if and only if it is a critical point of $\widehat{U}:=\left.U\right|_{\widehat{S}}$.

In the next result, we provide a representation of the Hessian quadratic form at any SBC w.r.t. to the the Euclidean as well as w.r.t. the mass scalar product.

Lemma 2.7 The Hessian of $\widehat{U}: \widehat{\mathbb{S}} \rightarrow \mathbb{R}$ at a critical point $q$ is the quadratic form on $T_{q} \widehat{\mathbb{S}}$ that can be represented w.r.t. the Euclidean and mass scalar product respectively by the dndimensional matrices

$$
\begin{aligned}
& \widehat{H}(q)=D^{2} U(q)+U(q) \widehat{S} M, \\
& H(q)=M^{-1} D^{2} U(q)+\widehat{S} U(q) .
\end{aligned}
$$

Remark 2.8 The latter representation is the natural choice if we are looking at the Hessian at an SBC as the linearization of the gradient flow $D \widetilde{\nabla} \widehat{U}(q)$, where $\widetilde{\nabla}$ is the gradient on $\mathbb{S}$ with respect to the Riemannian metric induced by the weighted mass-scalar product of the ambient manifold. 
By setting

$$
r_{i j}:=\left|q_{i}-q_{j}\right|, \quad u_{i j}:=\frac{q_{i}-q_{j}}{\left|q_{i}-q_{j}\right|}
$$

a direct computation shows that the $(i, j)$-entry of the block symmetric matrix $D^{2} U(q)$ is given by

$$
D_{i j}:=\frac{m_{i} m_{j}}{r_{i j}^{3}}\left[I_{d}-3 u_{i j} u_{i j}^{\top}\right] \text { for } i \neq j, \quad D_{i i}:=-\sum_{i \neq j} D_{i j} .
$$

The group $\mathrm{SO}(d)$ acts diagonally on $\widehat{\mathbb{S}}$. Since the potential is rotationally invariant, we get that

$$
D^{2} U(R q)=R^{\top} D^{2} U(q) R, \quad R \in \mathrm{SO}(d) .
$$

In particular, the Hessian of a $\mathrm{CC}$ is $\mathrm{SO}(d)$ invariant, whereas for general $\mathrm{SBC}$ the Hessian is invariant by a proper subgroup of $\mathrm{SO}(d)$, which is the trivial subgroup in case $s_{i} \neq s_{j}$ for all $i \neq j$.

\subsection{CSBC and Assumption (H1)}

In this subsection we provide a useful representation of the Hessian at a collinear SBC (CSBC in shorthand notation) and we discuss Assumption (H1) that will be used throughout the paper.

As a preliminary observation, we see from an easy inspection of Eq. (2.1) that the line containing each body $q_{k}$ of a CSBC must lie in an eigenspace of the matrix $S$. In particular, if the $s_{j}$ 's are pairwise distinct, then the only lines containing CSBC are the coordinate axis.

Notation 2.9 We can assume without loss of generality that each particle of the CSBC $q$ is in the " $j$ th eigenspace" $\left\langle e_{j}\right\rangle$ of $S$, where as usual $e_{j}$ denotes the $j$ th vector of the canonical basis. Up to relabeling the $e_{j}$ 's if necessary, we can assume that each body $q_{k}$ of the CSBC $q$ lies on $\mathbb{R} \times(0)^{d-1} \subset \mathbb{R}^{d}$, and so the unit vectors $u_{i j}$ are all multiples of $e_{1}$.

By a straightforward computation we get that each block $D_{i j}$ has the following representation

$$
D_{i j}=\frac{m_{i} m_{j}}{\left|r_{i j}\right|^{3}}\left[\begin{array}{cccc}
-2 & 0 & \ldots & 0 \\
0 & 1 & \ldots & 0 \\
\vdots & \vdots & \vdots & 0 \\
0 & 0 & \ldots & 1
\end{array}\right], \quad D_{i i}=-\sum_{j \neq i} D_{i j}
$$

After rearranging the $d n$ coordinates of the configuration vector $q$ by collecting the components into groups of $n$ variables with all of the $e_{1}$ components first, the $e_{2}$ components second and so on, the Hessian at the CSBC is of the form

$$
H(q)=\left[\begin{array}{llll}
-2 M^{-1} B(q) & & & \\
& M^{-1} B(q) & & \\
& & \ddots & \\
& & & M^{-1} B(q)
\end{array}\right]+\left[\begin{array}{llll}
s_{j} U(q) I_{n} & & & \\
& s_{1} U(q) I_{n} & & \\
& & \ddots & \\
& & & U(q) I_{n}
\end{array}\right]
$$


where $B(q)$ is the $n \times n$ matrix whose $(i, j)$-entry is given by

$$
b_{i i}(q)=-\sum_{\substack{j=1 \\ i \neq j}}^{n} \frac{m_{i} m_{j}}{r_{i j}^{3}}, \quad b_{i j}(q)=\frac{m_{i} m_{j}}{r_{i j}^{3}} .
$$

Remark 2.10 We recall that the dimension of the configuration sphere is $N-1=d(n-$ 1 ) $-1=\operatorname{dim} T_{q} \mathbb{S}$ and so, for $d=2$ (planar case) we get $2 n-3$, whereas for $d=3$ (spatial case), we get $3 n-4$. In the particular case of CCC (corresponding to $s_{1}=\ldots=s_{d}=1$ ), the $(n \times n)$-block matrix $M^{-1} B(q)$ has Morse index $(n-1)$ (this result can be traced back to Conley) with largest eigenvalue $-U(q)$, and nullity 1 with corresponding eigenvector $(1, \ldots, 1)$ (being transverse to the tangent space at $q$ to $\mathbb{S}$ ) which does not contribute to the inertia indices. Similarly, the block $-2 M^{-1} B(q)$ has Morse coindex $(n-1)$ and nullity 1 , which once again does not contribute to the inertia indices. In particular, the inertia indices of the CCC $q$ are

$$
\left(\iota^{-}(q), \iota^{0}(q), \iota^{+}(q)\right)=((d-1)(n-2), d-1, n-2) .
$$

Notice that $(d-1)(n-2)+(d-1)+(n-2)=d(n-1)-1=\operatorname{dim} T_{q} \mathbb{S}$.

Starting from Remark 2.10, in the next subsection we provide some interesting information about the inertia indices of CSBC which will be then the starting point for the lower bounds for the number of SBC that we will prove in Sect. 4. Hereafter, until the end of Sect. 4 we will always implicitly assume that

$$
s_{1}>s_{2}>\ldots>s_{d-1}>s_{d}=1 .
$$

Such an assumption might appear at a first glance rather restrictive. However, it has several noteworthy advantages that we will exploit throughout the paper and that we now thoroughly discuss. Before commenting further on Assumption (H1), we shall notice that there is no loss of generality in assuming that the $s_{i}$ 's be strictly decreasing and that $s_{d}=1$. This follows from the fact that we can always rearrange the vectors of the standard basis of $\mathbb{R}^{d}$ and that the problem is invariant under scaling.

- Assumption (H1) "kills" all symmetries of Eq. (2.1), thus allowing to apply standard Morse theory arguments (rather than more complicated theories, such as e.g. equivariant Morse theory or Morse-Bott theory) when looking for lower bounds on the number of SBC.

- The lower bounds on the number of SBC that one obtains assuming (H1) still allow to give (possibly not optimal but still remarkable) lower bounds on the number of SBC in the general case via a suitable "reduction to (H1) argument" as we now explain. Thus, suppose that for $d \geq 2$ positive real numbers $s_{1}>\ldots>s_{d}=1$ and positive integers $\mu_{1}, \ldots, \mu_{d} \in \mathbb{N}$ are given. On $\mathbb{R}^{D}, D:=\mu_{1}+\ldots+\mu_{d} \geq d$, we consider the $S$-balanced configurations problem with

$$
\widetilde{S}:=\operatorname{diag}(\underbrace{s_{1}, \ldots, s_{1}}_{\mu_{1} \text {-times }}, \ldots, \underbrace{s_{d}, \ldots, s_{d}}_{\mu_{d} \text {-times }}) .
$$

Notice that, under the assumptions above, Eq. (2.1) is invariant by the diagonal action on the configuration space of the subgroup of $\mathrm{SO}(D)$ given by the product group $\mathrm{SO}\left(\mu_{1}\right) \times$ 
$\ldots \times \mathrm{SO}\left(\mu_{d}\right)$. For each $i=1, \ldots, d$ we fix a line $L_{i}$ on the $\mu_{i}$-dimensional subspace of $\mathbb{R}^{D}$ relative to $s_{i}$, and restrict our attention to SBC which are contained in

$$
Y:=\bigoplus_{i=1}^{d} L_{i} \cong \mathbb{R}^{d} .
$$

Ignoring all vanishing components of configuration vectors which lie in $Y$, we obtain that SBC which are contained in $Y$ satisfy Eq. (2.1) on $\mathbb{R}^{d}$ with

$$
S:=\operatorname{diag}\left(s_{1}, \ldots, s_{d}\right),
$$

which is nothing else but (H1) on a proper subspace of $\mathbb{R}^{D}$. We call this procedure the REDUCTION TO (H1) ARGUMENT.

- Applying equivariant theories for the $S$-balanced configurations problem in $\mathbb{R}^{D}$ seems hopeless in full generality due-among other facts - to the amount of cases that one should treat (corresponding to different choices of the $\mu_{i}$ 's) and to the fact that, for $d \geq 3$, the diagonal action of the symmetry group $\mathrm{SO}\left(\mu_{1}\right) \times \ldots \times \mathrm{SO}\left(\mu_{d}\right)$ of Eq. (2.1) on the configuration space is not free, thus yielding a quotient which is not a manifold (actually, not even an orbifold).

- Theories like Morse-Bott theory, which allow to deal with functions having "nondegenerate" critical manifolds rather than critical points, do not take into account the symmetries of the problem and thus necessarily lead to weaker results. For instance, it is possible to show that for the $S$-balanced configurations problem on $\mathbb{R}^{4}$ with $S=\operatorname{diag}\left(s_{1}, s_{1}, 1,1\right)$, the lower bounds on the number of SBC that one obtains via the reduction to (H1) argument are precisely twice as much as the lower bounds that one obtains by Morse-Bott theory.

- All methods involving equivariant or stratified Morse theories are essentially based on equivariant homology and intersection homology theories, which are not computationally feasible in all cases.

- If the singular set consists only of collinear configurations, under suitable assumptions, one could try to overcome these difficulties by quotienting out the configuration space by the group action after removing the singular set. We will say more about this in Sect. 6, where we will compare the lower bounds on the number of SBC in $\mathbb{R}^{4}$ with $S=\operatorname{diag}\left(s_{1}, s_{1}, 1,1\right)$ obtained implementing such a strategy with the ones obtained via the reduction to (H1) argument (thus, by considering the $S$-balanced configurations problem in $\mathbb{R}^{2}$ with matrix $\left.\operatorname{diag}\left(s_{1}, 1\right)\right)$.

- Even if one is able to give lower bounds on the number of SBC not assuming (H1), for instance via equivariant Morse theory or Morse-Bott theory, one has additionally to show that the SBC that one finds are actually not CC. Notice indeed that, for SBC which are contained in a subspace of $\mathbb{R}^{D}$ corresponding to some $s_{i}$, Eq. (2.1) reduces to the central configurations equation. This is the same problem that one has to face when trying to exclude that spatial CC are actually not planar: despite the lower bounds provided by Pacella [11], it is so far not known whether there is more than one spatial CC which is not planar.

Assuming (H1), instead, we easily overcome this problem: indeed, under Assumption (H1), every solution of (2.1) which is not collinear cannot be a CC.

- Under Assumption (H1), solutions of Eq. (2.1) yield relative equilibria for the $n$-body problem in $\mathbb{R}^{2 d}$ via "complexification", which can be thought of as the inverse procedure to the reduction to (H1) argument. Indeed, $\operatorname{SBC}$ on $\mathbb{R}^{d}$ for $S=\operatorname{diag}\left(s_{1}, s_{2}, \ldots, s_{d-1}, 1\right)$ are in particular SBC in $\mathbb{R}^{2 d} \cong \mathbb{C}^{d}$ with matrix diag $\left(s_{1}, s_{1}, s_{2}, s_{2}, \ldots, s_{d-1}, s_{d-1}, 1,1\right)$. 


\subsection{CSBC and Corresponding CCC: Spectra and Inertia Indices}

In what follows, we refer to a CSBC in which every particle $q_{k}$ belongs to $\left\langle e_{j}\right\rangle$ (the eigenspace corresponding to the eigenvalue $s_{j}$ ) just as $s_{j}-\mathrm{CSBC}$. In this subsection, after describing the relation between $s_{j}-\mathrm{CSBC}$ and the corresponding $\mathrm{CCC}$, we provide an explicit description of their inertia indices.

Lemma 2.11 Let q be a $s$-CSBC, where $s=s_{j}$ for some $j=1, \ldots, d$. Then:

- $\widehat{q}:=\sqrt{s} q$ is a CCC.

- $M^{-1} B(q)+s U(q) I_{n}=s \sqrt{s}\left[M^{-1} B(\widehat{q})+U(\widehat{q}) I_{n}\right]$.

Proof By assumption, the CSBC $q$ lies in the eigenspace of $S$ corresponding to the eigenvalue $s$ and $|\sqrt{s} q|_{M}=1$. Thus, by a direct computation, we get

$$
\nabla U(\sqrt{s} \cdot q)=s^{-1} \nabla U(q)=-s^{-1} s U(q) M q=-U(q) M q=-U(\sqrt{s} \cdot q) M \sqrt{s} \cdot q .
$$

Setting $\widehat{q}:=\sqrt{s} \cdot q$, we obtain $\nabla U(\widehat{q})=-U(\widehat{q}) M \widehat{q}$ which implies that $\widehat{q}$ is a CCC. The homogeneity of $U$ implies that

$$
B(q)=s \sqrt{s} \cdot B(\widehat{q})
$$

and hence the eigenvalues of $B(q)$ are easily obtained by multiplying each eigenvalue of $B(\widehat{q})$ by $s \sqrt{s}$.

Remark 2.12 As we shall see below, from Lemma 2.11 it follows that the nullity of $q$ is generically zero, but might be non-zero for certain choices of the $s_{i}$ 's. Moreover, the coindex of a CSBC can possibly be greater than the coindex of the corresponding $\mathrm{CCC}$, as it could increase (but not decrease) for varying $s$. Analogously, the index of a CSBC can possibly be lower than the index of the corresponding CCC, as it could decrease (but not increase) for varying $s$. Loosely speaking, since the eigenvalues of $S$ are assumed to be greater than 1 , we get that adding to the block matrix $M^{-1} B(q)$ a block diagonal matrix of the form $s_{i} U(q) I_{n}$, we can move part of the spectrum located on the negative real line to the positive thus changing the inertia indices.

Another important property which is pointed out by Lemma 2.13 is the different role (with respect to the inertia indices of a CSBC) played by the different eigenspaces. In fact, for an $s-\mathrm{CSBC}$ the index is maximal, and the coindex is minimal, for $s=s_{1}$.

Lemma 2.13 Let $q$ be a CSBC. The following facts hold.

1. The index of $q$ is at most $(d-1)(n-1)$ and the coindex of $q$ is at least $(n-2)$

2. If $q$ is an $s_{1}-\mathrm{CSBC}$, then the Morse index is precisely $(d-1)(n-1)$ and the Morse coindex is precisely $n-2$. Furthermore, if $q$ is an $s_{j}-\mathrm{CSBC}$ and $j<d$, then $q$ cannot be a local minimum of $\widehat{U}$.

Proof We assume that $q$ is a $s_{j}$-CSBC for some $j \in\{1, \ldots, d\}$. By using Lemma 2.11, for $i \neq j$ we get that

$$
\begin{aligned}
M^{-1} B(q)+s_{i} U(q) I_{n} & =s_{j} \sqrt{s_{j}}\left[M^{-1} B(\widehat{q})+\left(\frac{s_{i}}{s_{j}}\right) U(\widehat{q}) I_{n}\right] \\
& =s_{j} \sqrt{s_{j}}\left[M^{-1} B(\widehat{q})+U(\widehat{q}) I_{n}+U(\widehat{q})\left(\frac{s_{i}-s_{j}}{s_{j}}\right) I_{n}\right]
\end{aligned}
$$


where $\widehat{q}$ is the CCC corresponding to $q$. Depending on $i$ and $j$, the quantity

$$
\left(\frac{s_{i}-s_{j}}{s_{j}}\right)
$$

can be positive or negative. For $j=1$ (corresponding to one of the two extremal cases), this term is always negative (i.e. independent of $i$ ). Thus, also the nullity of the central configuration $\widehat{q}$ contributes to the Morse index of the $s_{1}$-CSBC (in fact, the contribution is 1 for each of the $(d-1)$ blocks). The contribution to the coindex coming from the first block of the Hessian is $n-2$. Arguing analogously, we see that for $j>1$ the Morse index is strictly less than $(d-1)(n-1)$, whereas the coindex is strictly greater than $n-2$. This follows from the fact that, for $i<j$, the above quantity is strictly positive and hence the nullity of the CC $\widehat{q}$ contributes for such $i$ 's to the Morse coindex. Finally, for $j<d$, the above quantity for $i=d$ is strictly negative, thus the nullity of $\widehat{q}$ contributes to the Morse index.

The next result describes the properties of a CSBC in terms of the spectra of the corresponding CCC (or $s_{d}-\mathrm{CSBC}$ which is the same) as well as of the eigenvalues of $S$.

Lemma 2.14 Let $\widehat{q}$ be an $s_{d}-\mathrm{CSBC}$, and let

$$
\eta:=\text { smallest eigenvalue of } M^{-1} B(\widehat{q}) \text {. }
$$

Then:

- $\widehat{q}$ is a local minimum of $\widehat{U}$ provided that $\eta+s_{d-1} U(\widehat{q})>0$

- $\widehat{q}$ has non-vanishing Morse index if and only if $\eta+s_{d-1} U(\widehat{q})<0$.

- Suppose $\eta+s_{1} U(\widehat{q})<0$. For every $j=1, \ldots, d$, let $\widehat{q}_{j}$ be the $s_{j}$-CSBC whose ordering of the masses coincides with the one of $\widehat{q}$. Then, for any coordinate subspace $e^{1} \subset \mathbb{R}^{d}$ such that $\left\langle e_{j}\right\rangle \subset E, \operatorname{dim} E \geq 2$, we have that $\widehat{q}_{j}$ is not a local minimum of $\left.\widehat{U}\right|_{\widehat{\mathbb{S}} \cap E^{n}}$.

Proof For $i<d$, the smallest eigenvalue of the $i$ th block of the Hessian

$$
M^{-1} B(\widehat{q})+s_{i} U(\widehat{q}) I_{n}
$$

is given by

$$
\eta+s_{i} U(\widehat{q}) .
$$

The first two claims readily follows. As far as the last claim is concerned, we observe that for the $s_{j}-\mathrm{CSBC} \widehat{q}_{j}$ whose ordering of the masses coincides with the one of $\widehat{q}$ and for every $i \neq j$ we have that the corresponding block of the Hessian of $U$ at $\widehat{q}_{j}$ is given by

$$
M^{-1} B\left(\widehat{q}_{j}\right)+s_{i} U\left(\widehat{q}_{j}\right) I_{n}=s_{j} \sqrt{s}_{j}\left[M^{-1} B(\widehat{q})+\left(\frac{s_{i}}{s_{j}}\right) U(\widehat{q}) I_{n}\right] .
$$

In particular, the smallest eigenvalue of $M^{-1} B\left(\widehat{q}_{j}\right)+s_{i} U\left(\widehat{q}_{j}\right) I_{n}$ is (up to the positive factor $s_{j} \sqrt{s_{j}}$ ) given by

$$
\eta+\frac{s_{i}}{s_{j}} U(\widehat{q})
$$

We now estimate

$$
\eta+\frac{s_{i}}{s_{j}} U(\widehat{q})<\eta+\frac{s_{1}}{s_{j}} U(\widehat{q})<\eta+\frac{s_{1}}{s_{d}} U(\widehat{q})=\eta+s_{1} U(\widehat{q})<0,
$$

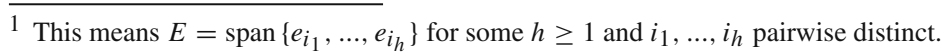


thus showing that the considered block has at least one negative eigenvalue. The claim readily follows.

Clearly, the value of $U(\widehat{q})$, as well as the matrix $B(\widehat{q})$ and its eigenvalues, depend on the choice of the CCC (i.e. on the choice of the ordering of the masses), unless all masses are equal. Therefore, the conditions above are a priori different for different ordering of the masses, and there is in general no direct way of comparing the inertia indices of CSBC with different ordering of the masses. Nevertheless, we can still use this information to deduce existence of non-collinear SBC as we now show.

Corollary 2.15 Under the notation above, if all masses are equal and

$$
1<s_{1}<-\frac{\eta}{U(\widehat{q})}
$$

then there are at least $\frac{d(d-1)}{2}$ planar non-collinear SBC. In particular, the $n$-body problem in $\mathbb{R}^{2 d} \cong \mathbb{C}^{d}$ has at least $\frac{d(d-1)}{2}$ continua of relative equilibria in which periodic motions form a dense subset. If the masses are not all equal, then the same conclusion holds provided one replaces the spectral condition above with

$$
1<s_{1}<\min \left\{-\frac{\eta}{U(\widehat{q})} \mid \widehat{q} \mathrm{CCC}\right\} .
$$

Proof For $1 \leq i<j \leq d$ fixed, we consider the $\left(e_{i}, e_{j}\right)$-plane in $\mathbb{R}^{d}$. If all masses are equal, then Lemma 2.14 implies that the global minimum of $\widehat{U}$ restricted to $\operatorname{span}\left\{e_{i}, e_{j}\right\}$ cannot be attained at a CSBC, and hence must be attained at a planar non-collinear SBC. The first part of the claim follows, since the cardinality of the set $\{(i, j) \mid 1 \leq i<j \leq d\}$ is precisely $\frac{d(d-1)}{2}$. We now set

$$
\mathcal{S}:=\left\{\left(s_{1}, \ldots, s_{d-1}\right) \mid-\frac{\eta}{U(\widehat{q})}>s_{1}>s_{2}>\ldots>s_{d-1}\right\} .
$$

The stability property of minima implies that the maps

$$
\left.\mathcal{S} \ni\left(s_{1}, \ldots, s_{d-1}\right) \mapsto \min \widehat{U}\right|_{\operatorname{span}\left\{e_{i}, e_{j}\right\}}, \quad 1 \leq i<j \leq d,
$$

yield the desired continua of relative equilibria. Such relative equilibria will be periodic motions whenever $s_{1}, \ldots, s_{d-1}, 1$ are rationally dependent, and quasi-periodic motions otherwise. This completes the proof in the case all masses are equal. The proof is analogous in the case of masses which are not all equal. Indeed, the new spectral gap condition implies that none of the CSBC can be a local minimum for the restriction of $\widehat{U}$ to $\operatorname{span}\left\{e_{i}, e_{j}\right\}$ as well. The details are left to the reader.

Remark 2.16 Corollary 2.15 holds for any choices of the masses $m_{1}, \ldots, m_{n}$, that is, independently of the fact that SBC are degenerate or not. For $n=3$ and $m_{1}=m_{2} \neq m_{3}$, one retrieves the quasi-periodic motions corresponding to isosceles triangles in a prescribed $\left(e_{i}, e_{j}\right)$-plane. (Cfr. [9, P.125], for further details).

Actually, by using similar arguments, it is possible to prove the existence of others continua of relative equilibria coming from minmax points (that must exist for topological reasons).

Remark 2.17 To any planar non-collinear CC we can associate two integers, the so-called planar index and normal index. As shown by Moeckel in [10, P. 69] (by using an argument 
originally due to Pacella) estimating from below the trace of $-M^{-1} B(q)$ at such a planar non-collinear $\mathrm{CC}$, it is possible to provide a lower bound on the normal index of a central configuration. A direct consequence of this estimate is that the global minimum of $\widehat{U}$ doesn't occur at a planar CC, and so proving the existence of at least one non-planar CC. It would be interesting to understand if an analogous argument can be carried over to the case of planar non-collinear SBC.

We conclude this section by determining the inertia indices of CSBC in case $d=2$. Thus, let $S=\operatorname{diag}\left(s_{1}, 1\right)$ with $s_{1}>1$. By Lemma 2.13 we get that the inertia indices of a $s_{1}-\mathrm{CSBC}$, denoted $q$, are

$$
\iota^{0}(q)=0, \quad \iota^{+}(q)=n-2, \quad \iota^{-}(q)=n-1,
$$

whereas for a $s_{2}-$ CSBC (which are nothing else but CCC, thus denoted $\widehat{q}$ ) we have

$$
\iota^{0}(\widehat{q}) \geq 0, \quad \iota^{+}(\widehat{q}) \geq n-2, \quad \iota^{-}(\widehat{q}) \leq n-1 .
$$

In the next result we provide a complete characterization of the inertia indices of $s_{2}-\mathrm{CSBC}$ depending on $s_{1}$ and on the spectrum of $M^{-1} B$ at $\widehat{q}$. Thus, let $\widehat{q}$ be a $s_{2}-$ CSBC (or, equivalently, a CCC), and denote by

$$
\eta_{k}(\widehat{q})<\ldots<\eta_{1}(\widehat{q})<-U(\widehat{q})<0
$$

the eigenvalues of the matrix $M^{-1} B(\widehat{q})$. Even though the $\eta_{i}$ 's depend on the choice of $\widehat{q}$, to ease the notation we hereafter drop the dependence on $\widehat{q}$. It is well known that the eigenvalues $-U(\widehat{q})$ and 0 are simple, but any other eigenvalue might have multiplicity. For $j=1, \ldots, k$ we denote by $\alpha_{j}$ the multiplicity of $\eta_{j}$ as an eigenvalue of $M^{-1} B(\widehat{q})$. We also set $\eta_{0}:=-U(\widehat{q})$ and $\alpha_{0}:=1$.

Proposition 2.18 Let $\widehat{q}$ be an $s_{2}-$ CSBC. Then, the following hold.

1. If $-\eta_{j}<s_{1} U(\widehat{q})<-\eta_{j+1}$ for some $j \in\{0, \ldots, k-1\}$, then

$$
\iota^{0}(\widehat{q})=0, \quad \iota^{+}(\widehat{q})=n-2+\sum_{i=0}^{j} \alpha_{i}, \quad \iota^{-}(\widehat{q})=n-1-\sum_{i=0}^{j} \alpha_{i} .
$$

2. If $s_{1} U(\widehat{q})=-\eta_{j}$ for some $j \in\{1, \ldots, k\}$, then

$$
\iota^{0}(\widehat{q})=\alpha_{j}, \quad \iota^{+}(\widehat{q})=n-2+\sum_{i=0}^{j-1} \alpha_{i}, \quad \iota^{-}(\widehat{q})=n-1-\sum_{i=0}^{j} \alpha_{i} .
$$

In particular, in this case, $\widehat{q}$ is a degenerate critical point of $\widehat{U}$.

3. If $s_{1} U(\widehat{q})+\eta_{k}>0$, then

$$
\iota^{0}(\widehat{q})=0, \quad \iota^{+}(\widehat{q})=2 n-3, \quad \iota^{-}(\widehat{q})=0 .
$$

In particular, in this case, $\widehat{q}$ is a local minimum of $\widehat{U}$.

Proof In order to prove the first claim we start observing that by adding the term $s_{1} U(\widehat{q}) I_{n}$ to the matrix $M^{-1} B(\widehat{q})$ part of the spectrum becomes positive. Since the assumption is equivalent to $\eta_{j+1}<-s_{1} U(\widehat{q})<\eta_{j}$, we get

$$
\eta_{j+1}+s_{1} U(\widehat{q})<0<\eta_{j}+s_{1} U(\widehat{q}) .
$$


So, to the Morse coindex of $q$ we add the contribution provided by the first $(j+1)$ eigenvalues of $M^{-1} B(\widehat{q})$ which become positive by adding $s_{1} U(\widehat{q})$. Clearly, the same contribution has to be subtracted from the Morse index. The proof of the second item is completely analogous. We just need to observe that the $(j+1)$ th eigenvalue $\eta_{j}$ of $M^{-1} B(\widehat{q})$ gives a non-trivial contribution to the nullity. The third item follows straightforwardly since, under the assumption $s_{1} U(\widehat{q})+\eta_{k}>0$, the whole spectrum of $M^{-1} B(\widehat{q})$ moves into the positive real axis.

Remark 2.19 As a direct consequence of Proposition 2.18, we get that there are only finitely many values of $s_{1}$ for which $s_{2}-\mathrm{CSBC}$ can be degenerate as critical points of $\widehat{U}$. In other words, $s_{2}-\mathrm{CSBC}$ are generically non-degenerate. We shall however remark that the values of $s_{1}$ that make an $s_{2}-\mathrm{CSBC}$ degenerate depend on the $s_{2}-\mathrm{CSBC}$, unless the masses are all equal. In a similar vein, where and how big the jumps on the Morse index and coindex are depend on the $s_{2}-\mathrm{CSBC}$.

A result analogous to Proposition 2.18, which will be omitted to keep the exposition as simple as possible, can be proved also for $d>2$, thus showing as a corollary that CSBC are generically non-degenerate.

\section{Estimates on the Coefficients of the Poincaré Polynomial}

In this section we provide some elementary but rather not trivial (asymptotic) estimates on the coefficients of the Poincaré polynomial of the collision free configuration sphere. The interest in such estimates relies mainly on the following facts:

- They yield non-trivial lower bounds for the number of SBC, since they show that also $s_{j}-\mathrm{CSBC}, j \geq 2$, can contribute to the count of critical points.

- They have highly non-trivial and rather unexpected qualitative consequences on the count of critical points. Indeed, in "some cases" $s_{j}-$ CSBC, $j \geq 2$, will contribute more than $s_{1}$-collinear SBC (this should be compared with [9, P. 151]).

- They might have applications in the study of limiting problems (i.e. with very large number of bodies).

We start recalling the Poincaré polynomial of the collision free configuration sphere $\widehat{\mathbb{S}}$. As this result is well-known, its proof will be omitted.

Lemma 3.1 The Poincaré polynomial of $\widehat{\mathbb{S}}$ is given by

$$
P(t)=\left(1+t^{d-1}\right)\left(1+2 t^{d-1}\right) \cdot \ldots \cdot\left(1+(n-1) t^{d-1}\right) .
$$

We are now ready to prove some results about the growth rate for the coefficient $c_{j}^{(n)}$ with $j=0, \ldots, n-1$ of the polynomial

$$
p_{n}(z):=(1+z)(1+2 z) \cdot \ldots \cdot(1+(n-1) z), \quad n \in \mathbb{N} .
$$

It is worth observing that the Poincare polynomial $P(t)$ of $\widehat{\mathbb{S}}$ is nothing else but $p_{n}\left(t^{d-1}\right)$. For notational convenience we hereafter $\operatorname{set} c_{j}^{(n)}=0$ for every $j \geq n$.

Proposition 3.2 The following statements hold:

1. $\sum_{j=0}^{n-1} c_{j}^{(n)}=n !$ for every $n \in \mathbb{N}$ 
2. $c_{j}^{(n)} \leq \frac{n !}{2}$ for every $j \in \mathbb{N}_{0}$ and every $n \geq 2$

3. For fixed $j \in \mathbb{N}_{0}$, we get

$$
\lim _{n \rightarrow+\infty} \frac{c_{j}^{(n)}}{n !}=0
$$

4. For every $n \in \mathbb{N}$, we get

$$
c_{n-1}^{(n)}=(n-1) ! \text { and } c_{n-2}^{(n)} \sim(\gamma+\log n)(n-1) !
$$

where

$$
\gamma:=\lim _{n \rightarrow+\infty}\left(\sum_{j=1}^{n} \frac{1}{j}-\log n\right)
$$

is the Euler-Mascheroni constant. More generally, for every fixed $j \in \mathbb{N}$ we get

$$
c_{n-j}^{(n)} \lesssim(\gamma+\log n)^{j-1}(n-1) !
$$

where “¿” means inequality up to some constant independent on $j$ and $n$. Hence, in particular

$$
\lim _{n \rightarrow+\infty} \frac{c_{n-j}^{(n)}}{n !}=0 .
$$

Remark 3.3 Part 1 of Proposition 3.2 yields the following interesting representation of the factorial of $n$ :

$$
n !=1+\sum_{i_{1}=1}^{n-1} i_{1}+\sum_{1 \leq i_{1}<i_{2} \leq n-1} i_{1} i_{2}+\ldots+\sum_{1 \leq i_{1}<\ldots<i_{n-2} \leq n-1} i_{1} \cdot \ldots \cdot i_{n-2}+(n-1) !
$$

To our best knowledge, such a representation has never appeared in the literature. Notice also that Part 1 immediately implies that all but at most one coefficients $c_{j}^{(n)}$ are smaller than $n ! / 2$. We observe that the inequality in Part 2 is strict as soon as $n \geq 4$.

\section{Proof}

1. The claim follows trivially by evaluating

$$
p_{n}(z)=(1+z)(1+2 z) \cdot \ldots \cdot(1+(n-1) z)=\sum_{j=0}^{n-1} c_{j}^{(n)} z^{j}
$$

at $z=1$. However we provide a proof by induction over $n \in \mathbb{N}$. For $n=1$ the claim is obvious. Now, suppose the claim holds true for $n$. Since

$$
\sum_{j=0}^{n} c_{j}^{(n+1)} z^{j}=(1+n z) \sum_{j=0}^{n-1} c_{j}^{(n)} z^{j}
$$

evaluating at $z=1$ yields by inductive assumption

$$
\sum_{j=0}^{n} c_{j}^{(n+1)}=\sum_{j=0}^{n-1} c_{j}^{(n)} z^{j}+n \sum_{j=0}^{n-1} c_{j}^{(n)} z^{j}=n !+n n !=(n+1) n !=(n+1) !
$$


2. Once again by induction over $n$. For $n=2$ the claim is trivially satisfied. Assuming that the claim hold for $n$ yields for every $j \in \mathbb{N}_{0}$ :

$$
c_{j}^{(n+1)}=n c_{j-1}^{(n)}+c_{j}^{(n)} \leq n \frac{n !}{2}+\frac{n !}{2}=\frac{(n+1) !}{2} .
$$

3. By induction over $j \in \mathbb{N}_{0}$. The claim is obvious for $j=0$ as $c_{0}^{(n)}=1$ for all $n \in \mathbb{N}_{0}$. Now, suppose that the claim hold for $j$. Then, for $\epsilon>0$ there exists $n_{0}=n_{0}(\epsilon) \in \mathbb{N}$ such that $c_{j}^{(n)} / n !<\epsilon$ for every $n \geq n_{0}$, and hence using 2 . we get for every $n \geq \max \left\{n_{0}, \frac{1}{2 \epsilon}-2\right\}$ :

$$
\begin{aligned}
\frac{c_{j+1}^{(n+1)}}{(n+1) !} & =n \frac{c_{j}^{(n)}}{(n+1) !}+\frac{c_{j+1}^{(n)}}{(n+1) !} \\
& =\frac{n}{n+1} \frac{c_{j}^{(n)}}{n !}+\frac{c_{j+1}^{(n)}}{(n+1) !} \\
& <\frac{n}{n+1} \epsilon+\frac{1}{2(n+1)} \\
& <2 \epsilon .
\end{aligned}
$$

Remark 3.4 Alternatively, one can show that for $j \in \mathbb{N}$ fixed, the coefficient $c_{j}^{(n)}$ has a polynomial growth in $n$.

4. The first claim is trivial. As far as $c_{n-2}^{(n)}$ is concerned, we compute

$$
c_{n-2}^{(n)}=\sum_{i=1}^{n-1} 1 \cdot 2 \cdot \ldots \cdot \hat{i} \cdot \ldots \cdot(n-1)=(n-1) ! \cdot \sum_{i=1}^{n-1} \frac{1}{i} \sim(n-1) ! \cdot(\gamma+\log (n-1))
$$

and the claim follows. Now, for fixed $j \geq 2$ we compute

$$
\begin{aligned}
c_{n-j}^{(n)} & =\sum_{1 \leq i_{1}<\ldots<i_{j-1} \leq n-1} 1 \cdot \ldots \cdot \widehat{i}_{1} \cdot \ldots \cdot \widehat{i}_{j-1} \cdot \ldots \cdot(n-1) \\
& =(n-1) ! \sum_{1 \leq i_{1}<\ldots<i_{j-1} \leq n-1} \frac{1}{i_{1} \cdot \ldots \cdot i_{j-1}} \\
& =(n-1) ! \sum_{i_{1}=1}^{n-1-j} \frac{1}{i_{1}} \sum_{i_{2}=i_{1}+1}^{n-j} \frac{1}{i_{2}} \cdot \ldots \cdot \sum_{i_{j-1}=i_{j-2}+1}^{n-1} \frac{1}{i_{j-1}} \\
& \lesssim(n-1) ! \sum_{i_{1}=1}^{n-1-j} \frac{1}{i_{1}} \sum_{i_{2}=i_{1}+1}^{n-j} \frac{1}{i_{2}} \cdot \ldots \cdot \sum_{i_{j-2}=i_{j-3}+1}^{n-2} \frac{1}{i_{j-2}} \cdot(\gamma+\log (n-1)) \\
& \lesssim \ldots \\
& \lesssim(n-1) ! \prod_{k=1}^{j-1}(\gamma+\log (n-k)) \\
& <(n-1) !(\gamma+\log (n-1))^{j-1} .
\end{aligned}
$$


Finally, for fixed $j \in \mathbb{N}$

$$
\frac{c_{n-j}^{n}}{n !} \lesssim \frac{(n-1) !(\gamma+\log (n-1))^{j-1}}{n !}=\frac{(\gamma+\log (n-1))^{j-1}}{n} \rightarrow 0, \quad \text { as } n \rightarrow+\infty .
$$

Remark 3.5 For $j \in \mathbb{N}$ sufficiently large the estimate provided by the last item in Proposition 3.2 , is very far from being optimal. In fact, as we shall see below, a much stronger statement holds. More precisely, for every sequence $\left\{j_{n}\right\}_{n} \subset \mathbb{N}$ we have

$$
\lim _{n \rightarrow+\infty} \frac{c_{n-j_{n}-1}^{(n)}}{n !}=0 .
$$

The last two items of Proposition 3.2 confirm the validity of (3.3) when $\left\{n-j_{n}\right\}_{n}$ and $\left\{j_{n}\right\}_{n}$ are uniformly bounded. In the case of positively divergent sequences $j_{n}$, the behavior is described by Proposition 3.6.

Proposition 3.6 Let $\left\{j_{n}\right\}_{n} \subset \mathbb{N}$ be a positively divergent sequence. Then, the following hold.

1. For every $\epsilon>0$,

$$
\lim _{n \rightarrow+\infty} \frac{c_{n-j_{n}-1}^{(n)}}{n^{\epsilon}(n-1) !}=0 .
$$

In particular the asymptotic condition (3.3) holds.

2. If $j_{n} \gtrsim \log \log n$, then

$$
\lim _{n \rightarrow+\infty} \frac{c_{n-j_{n}-1}^{(n)}}{(n-1) !}=0 .
$$

3. If $j_{n} \gtrsim \log n$, then for every $k \in \mathbb{N}$ fixed

$$
\lim _{n \rightarrow+\infty} \frac{c_{n-j_{n}-1}^{(n)}}{(n-k) !}=0 .
$$

Remark 3.7 Proposition 3.6 states that every sequence $\left\{c_{k_{n}}^{(n)}\right\}_{n}$ of coefficients of the polynomials $p_{n}(z)$ grows slower than $n$ !. Moreover, if the sequence $\left\{k_{n}\right\}_{n}$ does not grow "too fast", then we get even a slower growth; in fact, $c_{k_{n}}^{(n)}$ grows slower than $(n-1)$ ! if $k_{n} \lesssim n-\log \log n$ and slower than $(n-k)$ ! for every $k \in \mathbb{N}$ provided $k_{n} \lesssim n-\log n$. Notice that for $\left\{k_{n}\right\}_{n}$ bounded we have polynomial growth, and that $(n-1)$ ! is precisely the coefficient of $p_{n}(z)$ of degree $(n-1)$.

Before proving the proposition we observe that for every $n \in \mathbb{N}$ and every $j \leq n-1$ :

$$
\begin{aligned}
c_{n-j-1}^{(n)} & =\sum_{1 \leq i_{1}<\ldots<i_{j} \leq n-1} 1 \cdot \ldots \cdot \widehat{i}_{1} \cdot \ldots \cdot \widehat{i}_{j} \cdot \ldots \cdot(n-1) \\
& =(n-1) ! \sum_{1 \leq i_{1}<\ldots<i_{j} \leq n-1} \frac{1}{i_{1} \cdot \ldots \cdot i_{j}} \\
& =(n-1) ! \sum_{i_{1}=1}^{n-j-1} \frac{1}{i_{1}} \cdot \sum_{i_{2}=i_{1}+1}^{n-j} \frac{1}{i_{2}} \cdot \ldots \cdot \sum_{i_{j}=i_{j-1}+1}^{n-1} \frac{1}{i_{j}}
\end{aligned}
$$




$$
\lesssim(n-1) ! \int_{1}^{n} \frac{1}{i_{1}} \cdot \int_{i_{1}}^{n} \frac{1}{i_{2}} \cdot \ldots \cdot \int_{i_{j-1}}^{n} \frac{1}{i_{j}} \mathrm{~d} i_{j} \cdot \ldots \cdot \mathrm{d} i_{1} .
$$

The key step is the following result, whose proof is postponed to Appendix A.

Lemma 3.8 For every $n, j \in \mathbb{N}$ we have

$$
\int_{1}^{n} \frac{1}{i_{1}} \cdot \int_{i_{1}}^{n} \frac{1}{i_{2}} \cdot \ldots \cdot \int_{i_{j-1}}^{n} \frac{1}{i_{j}} \mathrm{~d} i_{j} \cdot \ldots \cdot \mathrm{d} i_{1}=\frac{1}{j !} \log ^{j}(n) .
$$

\section{Proof of Proposition 3.6}

1. Using Eq. (3.4), Lemma 3.8, and Stirling's approximation of $n$ !, we get

$$
\begin{aligned}
\frac{c_{n-j_{n}-1}^{(n)}}{n^{\epsilon}(n-1) !} & \lesssim \frac{\log j_{n}(n)}{n^{\epsilon} j_{n} !} \\
& \sim \frac{e^{\log \left(j_{n} \cdot \log n\right)}}{n^{\epsilon} \sqrt{2 \pi j_{n}\left(\frac{j_{n}}{e}\right)^{j_{n}}}} \\
& =\frac{e^{\log \left(j_{n} \cdot \log n\right)}}{e^{\epsilon \log n+\frac{1}{2} \log \left(2 \pi j_{n}\right)+j_{n} \log j_{n}-j_{n}}} \\
& =e^{\log j_{n}+\log \log n-\epsilon \log n-\frac{1}{2} \log \left(2 \pi j_{n}\right)-j_{n} \log j_{n}+j_{n}},
\end{aligned}
$$

and we readily see that the exponent goes to $-\infty$ for $n \rightarrow+\infty$, as $\epsilon \log n$ dominates $\log \log n$ and $j_{n} \log j_{n}$ dominates the remaining terms.

2. Arguing in a similarly way, we finally get

$$
\frac{c_{n-j_{n}-1}^{(n)}}{(n-1) !} \lesssim \frac{\log j_{n}(n)}{j_{n} !} \sim e^{\log j_{n}+\log \log n-\frac{1}{2} \log \left(2 \pi j_{n}\right)-j_{n} \log j_{n}+j_{n}} \rightarrow 0,
$$

as $j_{n} \log j_{n}$ is the leading term for $j_{n} \gtrsim \log \log n$.

3. For fixed $k \geq 2$, we compute

$$
\begin{aligned}
\frac{c_{n-j_{n}-1}^{(n)}}{(n-k) !} & \lesssim(n-1) \cdot \ldots \cdot(n-k+1) \cdot \frac{\log ^{j_{n}}(n)}{j_{n} !} \\
& \sim e^{(k-1) \log n+\log j_{n}+\log \log n-\frac{1}{2} \log \left(2 \pi j_{n}\right)-j_{n} \log j_{n}+j_{n}} \rightarrow 0,
\end{aligned}
$$

as $j_{n} \log j_{n}$ is the leading term provided $j_{n} \gtrsim \log n$.

\section{Lower Bounds on the Number of SBC}

This section is devoted to prove lower bounds on the number of SBC under the additional assumption

(H2) All SBC are non-degenerate.

Such lower bounds follow from the estimates provided in Sect. 3 and the Morse inequalities. We briefly recall that the Morse inequalities of a Morse function $f$ on a closed manifold $\mathcal{M}$ relates the Morse indices of the (non-degenerate) critical points to the Betti numbers of 
the manifold. Usually, these inequalities are expressed in terms of polynomial generating function. More precisely, we define the MORSE POLYNOMIAL as

$$
M(t):=\sum_{k} \gamma_{k} t^{k} \quad \text { where } \gamma_{k} \text { is the number of critical points of } f \text { having index } k
$$

and the POINCARÉ POLYNOMIAL as

$$
P(t)=\sum_{k} \beta_{k} t^{k} \quad \text { where } \beta_{k} \text { denotes the } k \text { th Betti number of the manifold }
$$

i.e. the rank of the $k$ th homology group with real (or rational) coefficients. Then the Morse inequalities read

$$
M(t)=P(t)+(1+t) R(t)
$$

where $R(t)$ is a polynomial with non-negative integer coefficients. So, as a direct consequence of the positivity of the coefficients of $R(t)$ one obtains that $\beta_{k}$ is a lower bound for $\gamma_{k}$.

Remark 4.1 Even if the collision free configuration sphere $\widehat{\mathbb{S}}$ is not compact, we are still allowed to use the Morse inequalities to derive lower bounds on the number of SBC. Indeed, as it is well-known, $U(q) \rightarrow+\infty$ as $q$ approaches the singular set $\Delta$.

In Theorem 4.2 below we provide a lower bound on the number of planar SBC when all masses are equal. This lower bound depends on a spectral gap condition at any CCC, which is independent of the chosen CCC. Moreover, as a direct consequence of the Assumption (H1), non-collinear SBC cannot be CC.

As in Sect. $2, \eta_{k}<\ldots<\eta_{1}<-U(\widehat{q})<0$ denote the eigenvalues of the matrix $M^{-1} B(\widehat{q})$.

Theorem 4.2 Suppose that $m_{1}=m_{2}=\ldots=m_{n}$. Under the previous notation and Assumptions (H1) \& (H2), the following lower bounds on the number of planar SBC hold:

1. If $1<s_{1}<-\frac{\eta_{1}}{U(\widehat{q})}$ there are at least

$$
3 n !-2(n-1) !
$$

planar SBC, of which at least

$$
n !-2(n-1) !
$$

are not collinear.

2. For $-\frac{\eta_{1}}{U(\widehat{q})}<s_{1}<-\frac{\eta_{k}}{U(\widehat{q})}$ there are at least

$$
4 n !-2(n-1) !
$$

planar SBC of which at least

$$
2 n !-2(n-1) !
$$

are not collinear. Moreover, for $n \in \mathbb{N}$ sufficiently large, we get the following (improved) estimate

$$
\left(3 n-2\left(1+n^{\epsilon}\right)\right) \cdot(n-1) !
$$

non-collinear SBC for some constant $\epsilon:=\epsilon(n) \rightarrow 0$ for $n \rightarrow+\infty$. 
3. If $-\frac{\eta_{k}}{U(\widehat{q})}<s_{1}$ there are at least

$$
5 n !-2(n-1) !-2
$$

planar SBC, of which at least

$$
3 n !-2(n-1) !-2
$$

are not collinear.

Remark 4.3 For values of $s_{1}$ close to 1 we get worse lower bounds than in the other cases. This is not surprising, since in this case the Morse index of $s_{2}-\mathrm{CSBC}$ are precisely one less the Morse index of $s_{1}-\mathrm{CSBC}$, and hence cancellations in homology might occur.

By interpreting the parameter $s$ as a bifurcation parameter along the trivial branch of 1CSBC, the scenarios provided in Theorem 4.2 agree with the numbers of bifurcation instants as explained in [3, Theorem 4.7]. Moreover, in the final section of [3] the authors produce for the three body problem with equal masses an explicit description of this phenomenon as depicted in [3, Fig. 1]. In this respect, we also mention [2, Theorem 4] in which authors prove the existence of three smooth families of SBC as illustrated in Fig. 2 of the aforementioned paper.

Proof All statements are consequences of the estimates for the coefficients of the Poincaré polynomial proved in Sect. 3 and the Morse inequalities, which may be written using Eq. (4.3) as

$$
\sum_{i=0}^{n-1} \gamma_{i} z^{i}=p_{n}(z)+(1+z) R(z)=\sum_{i=0}^{n-1} c_{i}^{(n)} z^{i}+(1+z) \sum_{i=0}^{n-1} r_{i} z^{i},
$$

for some polynomial $R(z)$ having non-negative integer coefficients $r_{i}$, where $\gamma_{i}$ denotes the number of critical points of $\widehat{U}$ having Morse index $i$. Recall that the Morse index of $s_{1}-\mathrm{CSBC}$ is $n-1$, as it follows from Lemma 2.11, independently of $s_{1}$.

1. In virtue of Item 1 of Proposition 2.18 for $j=0$, the assumption yields that the Morse index of $s_{2}-\mathrm{CSBC}$ is $n-2$. In this case, we will simply ignore $s_{2}-\mathrm{CSBC}$. Applying the full Morse inequalities together with Proposition 3.2 yields

$$
\gamma_{n-1}=(n-1) !+r_{n-2}+r_{n-1} \geq n ! \quad \Rightarrow \quad r_{n-2}+r_{n-1} \geq n !-(n-1) !
$$

Therefore, evaluating the Morse inequalities at $z=1$, we get

$$
\begin{aligned}
\sum_{i=0}^{n-1} \gamma_{i}=p_{n}(1)+2 R(1) & =n !+2 \sum_{i=0}^{n-1} r_{i} \\
& \geq n !+2\left(r_{n-3}+r_{n-2}+r_{n-1}\right) \\
& \geq n !+2\left(r_{n-2}+r_{n-1}\right) \\
& \geq 3 n !-2(n-1) !
\end{aligned}
$$

Finally, we subtract $2 n$ ! which is the number of CSBC lying on the coordinate axes, which are the only possible CSBC in virtue of Assumption (H1), thus obtaining the desired lower bound. 
2. Under the given assumption, the Morse index $j$ of a $s_{2}-$ CSBC is strictly smaller than $n-2$ (in fact, at least $\eta_{1}$ moves into the positive line by adding $s_{1} U(\widehat{q})$ ). Applying again the full Morse inequalities together with Proposition 3.2 we obtain

$$
\begin{aligned}
\gamma_{n-1}=(n-1) !+r_{n-2}+r_{n-1} \geq n ! & \Rightarrow \quad r_{n-2}+r_{n-1} \geq n !-(n-1) ! \\
\gamma_{j}=c_{j}^{(n)}+r_{j-1}+r_{j} \geq n ! & \Rightarrow \quad r_{j-1}+r_{j} \geq n !-c_{j}^{(n)} \geq \frac{n !}{2}
\end{aligned}
$$

and hence

$$
\sum_{i=0}^{n-1} \gamma_{i} \geq n !+2\left(r_{j-1}+r_{j}+r_{n-2}+r_{n-1}\right) \geq 4 n !-2(n-1) !
$$

For $n \in \mathbb{N}$ sufficiently large enough we may replace (4.6) with

$$
r_{j-1}+r_{j} \geq n !-c_{j}^{(n)} \geq n !-n^{\epsilon}(n-1) !
$$

where $\epsilon$ is as given in Item 1 of Proposition 3.6. The claim follows.

3. In this case $s_{2}-\mathrm{CSBC}$ are local minima of $\widehat{U}$ and hence

$$
\gamma_{0}=c_{0}^{(n)}+r_{0}+r_{1} \geq n ! \Rightarrow r_{0}+r_{1} \geq n !-c_{0}^{(n)}=n !-1
$$

which yields the desired lower bound.

In the next result we apply the asymptotic estimates for the coefficients of the Poincaré polynomial proved in Sect. 3 to improve the lower bounds on the number of SBC for large values of $n$ in cases all masses are equal. The outcome will be that $s_{j}-\mathrm{CSBC}, j \geq 2$, asymptotically will - in many cases - give more contribution than the $s_{1}-\mathrm{CSBC}$ to the count of SBC. As already explained, the importance of such a result relies more in its qualitative rather than in its quantitative aspect, since such improvements will only be marginal. We recall that $\alpha_{j}$ denotes the multiplicity of the eigenvalue $\eta_{j}$ of $M^{-1} B(\widehat{q})$, where $\widehat{q}$ is any CCC of $n$ equal masses (cfr. Sect. 2.2 for further details).

Lemma 4.4 For $n \in \mathbb{N}$ large enough, we define $j_{n} \in\{1, \ldots, n\}$ such that

$$
\sum_{i=0}^{j_{n}-1} \alpha_{i}<\log n<\sum_{i=0}^{j_{n}} \alpha_{i}
$$

Assuming that $(H 1) \&(H 2)$ hold, for $-\frac{\eta_{j_{n}}}{U(\widehat{q})}<s_{1}$ there exist at least

$$
5 n !-2(n-1) !-2(n-h) !
$$

planar $\mathrm{SBC}$, for some $h \geq 1$, of which at least

$$
3 n !-2(n-1) !-2(n-h) !
$$

are not collinear.

Proof Under the assumptions, the Morse index $j$ of a $s_{2}-$ CSBC will be smaller than $n-\log n-1$. Using Item 3 of Proposition 3.6, we find an $h \geq 1$ such that

$$
c_{j}^{(n)} \leq(n-h) !
$$


and this yields by the Morse inequalities

$$
r_{j-1}+r_{j} \geq n !-(n-h) !
$$

The claim follows by arguing as in the proof of Theorem 4.2.

We now drop the condition that all masses be equal and derive lower bounds on the number of SBC assuming (H1) and (H2). As already observed, in this case the jumps on the Morse index of $s_{2}-\mathrm{CSBC}$ might occur at different values of $s_{1}$ for different orderings of the masses. Hence, a precise statement as in Theorem 4.2 is unfortunately no longer available. Imposing further conditions, one could still apply the asymptotic estimates proved in Sect. 3, thus obtaining better lower bounds. However, we refrain to do this in order to keep the exposition as reader friendly as possible. Notice also that, by the pidgeonhole principle, at least $(n-1)$ ! of the $s_{2}-$ CSBC must have the same Morse index; these will then contribute to the count of critical points provided the corresponding coefficient of the Poincaré polynomial be smaller than $(n-1)$ ! (which is, in virtue of the discussion in Sect. 3, asymptotically very likely to be the case).

Theorem 4.5 Under Assumptions (H1) and (H2), if

$$
s_{1}>\max \left\{-\frac{\eta_{k}}{U(\widehat{q})} \mid \widehat{q} \mathrm{CCC}\right\}
$$

then there are at least

$$
3 n !-2(n-1) !-2
$$

planar non-collinear SBC. Otherwise, there are at least

$$
n !-2(n-1) !
$$

planar non-collinear SBC.

Proof Under the given spectral gap assumption, we readily see that every $s_{2}-\mathrm{CSBC}$ is a local minimum of $\widehat{U}$. The claim follows as in Part 3 of Theorem 4.2. The other claim is obtained using the Morse inequalities only taking into account the contribution given by $s_{1}-$ CSBC. The details are left to the reader.

For $d>2$ the Poincaré polynomial is given by

$$
p_{n}\left(t^{d-1}\right)=\left(1+t^{d-1}\right)\left(1+2 t^{d-1}\right) \cdot \ldots \cdot\left(1+(n-1) t^{d-1}\right),
$$

and all coefficients in degree different from $j(d-1)$ vanish identically. In the next proposition, we provide lower bounds for the number of SBC in case of equal masses for some particular case: in the worst possible case, namely when the Morse indices of all CSBC differ precisely by one, in an intermediate case, namely when all Morse indices of CSBC differ by at least two, and in the best possible case, namely when the Morse indices of CSBC are not integer multiples of $(d-1)$ for all but the $s_{1}$-collinear SBC.

Proposition 4.6 For $m_{1}=\ldots=m_{n}$, under Assumptions (H1) \& (H2), the following statements hold:

1. Suppose that, for every $j=1, \ldots, d-1$, the Morse indices of $s_{j}-\mathrm{CSBC}$ and $s_{j+1}-\mathrm{CSBC}$ differ precisely by one. Then, there are at least

$$
\left(d+\frac{1}{2}\right) n !-(n-1) !
$$


SBC of which at least

$$
\frac{n !}{2}-(n-1) !
$$

are not collinear. For large values of $n \in \mathbb{N}$ the lower bound can be improved to

$$
(n-(1+\gamma+\log n)) \cdot(n-1) !
$$

non collinear $\mathrm{SBC}$

2. If, for every $j=1, \ldots, d-1$, the Morse indices of $s_{j}-\mathrm{CSBC}$ and $s_{j+1}-\mathrm{CSBC}$ differ at least by two, then there are at least

$$
2 n !-2(n-1) !
$$

non collinear SBC.

3. Suppose that, for every $j \geq 1$, the Morse indices of $s_{j}-\mathrm{CSBC}$ and $s_{j+1}-\mathrm{CSBC}$ differ at least by two, and that for every $j>1$ the Morse index is not an integer multiple of $(d-1)$. Then, there are at least

$$
(d+1) n !-2(n-1) !
$$

non collinear SBC.

\section{Proof}

1. In this case, the Morse indices of CSBC are precisely

$$
(d-1)(n-2),(d-1)(n-2)+1, \ldots,(d-1)(n-1),
$$

so that the corresponding coefficients of the Poincaré polynomial vanishes only for the smallest resp. largest Morse index. The full Morse inequalities and Proposition 3.2 yield now for the coefficients of the remainder $R(z)$ :

$$
\begin{aligned}
r_{(n-1)(d-1)-1}+r_{(n-1)(d-1)} & \geq n !-(n-1) ! \\
r_{(n-1)(d-1)-j-1}+r_{(n-1)(d-1)-j} & \geq n ! \quad j=1, \ldots, d-2, \\
r_{(n-2)(d-1)-1}+r_{(n-2)(d-1)} & \geq n !-c_{n-2}^{(n)} \geq \frac{n !}{2},
\end{aligned}
$$

and hence

$$
\sum_{j} \gamma_{j} \geq n !+(d-1) n !-(n-1) !+\frac{n !}{2}=\left(d+\frac{1}{2}\right) n !-(n-1) !
$$

The asymptotic estimate is obtained using again Item 3 of Proposition 3.2.

2. Denoting by $\mu_{j}$ the Morse index of $s_{j}-$ CSBC for $j>1$, from

$$
\begin{aligned}
r_{(n-1)(d-1)-1}+r_{(n-1)(d-1)} & \geq n !-(n-1) ! \\
r_{\mu_{j}-1}+r_{\mu_{j}} & \geq n !-c_{\mu_{j}}^{(n)} \geq \frac{n !}{2},
\end{aligned}
$$

we obtain

$$
\sum_{j} \gamma_{j} \geq n !+2(n !-(n-1) !)+(d-1) n !=(d+2) n !-2(n-1) !
$$


3. In this case we have

$$
r_{\mu_{j}-1}+r_{\mu_{j}} \geq n !-c_{\mu_{j}}^{(n)}=n ! \quad \forall j>1
$$

and hence

$$
\sum_{j} \gamma_{j} \geq n !+2(n !-(n-1) !)+2(d-1) n !=(2 d+1) n !-2(n-1) !
$$

In the proposition above we applied Morse theory on the whole collision free configuration sphere in $\mathbb{R}^{\text {nd }}$, and as we can see, in two of the three cases we obtain lower bounds independent of the dimension $d$. Implementing a strategy similar to the one used in Corollary 2.15, we can instead give a general lower bound (i.e. independent of the Morse indices of the CSBC as well as of the fact that the masses be equal or not) on the number of non-collinear SBC which grows quadratically in $d$.

Before doing that in the next theorem, we shall notice that Part 1 of the proposition above holds also for non-equal masses provided all $s_{j}$ are sufficiently close to one. Indeed, also in this case all $s_{j}-\mathrm{CSBC}$ have the same Morse index and the difference of Morse indices between $s_{j}-\mathrm{CSBC}$ and $s_{j+1}-\mathrm{CSBC}$ is always equal one. This shows that, at least for $s_{j}$ close to one, all CSBC contribute to the count of SBC, even if the masses are not all equal. Notice also that, ignoring $s_{j}-\mathrm{CSBC}$ for $j \geq 2$ (as done in Theorem 4.2) does not provide any non-trivial lower bound for $d>2$.

Theorem 4.7 For fixed $d \geq 2$, under Assumptions $(H 1) \&(H 2)$, there are at least

$$
\frac{d(d-1)}{2} \cdot(n !-2(n-1) !)
$$

planar non-collinear SBC.

Proof For all $1 \leq i<j \leq d$, the manifold $\mathcal{P}_{i, j}$ of configurations which are contained in the $\left(e_{i}, e_{j}\right)$-plane is invariant under the gradient flow of $\widehat{U}$. Therefore, we can do Morse theory on $\mathcal{P}_{i, j}$, which in turn is nothing else but the collision free configuration space in the plane. Notice indeed, that Assumptions (H1) and (H2) hold also for the restriction to $\mathcal{P}_{i, j}$. Therefore, using Theorem 4.5, we obtain that there are at least

$$
n !-2(n-1) !
$$

non-collinear SBC in the $\left(e_{i}, e_{j}\right)$-plane. Furthermore, Assumption (H1) implies that the symmetry group of Eq. (2.1) is trivial, and hence planar SBC contained in different planes can be counted separately. This together with the fact the number of different pairs $1 \leq i<j \leq d$ is precisely $d(d-1) / 2$ implies the claim.

Also in this case, we could obtain better lower bounds by a case distinction and implementing the asymptotic growth estimates for the coefficients of the Poincare polynomial. Again, we refrain to do this to obtain better readability. We finish this section noticing that information on the inertia indices of planar SBC might help to improve the above result by e.g. showing the existence of non-planar SBC. This is indeed object of ongoing research.

\section{The $45^{\circ}$ Theorem for Balanced Configurations}

The well-known $45^{\circ}$ - THEOREM for collinear CC is a "global version" of the fact that collinear $\mathrm{CC}$ are an attractor for the projectivized gradient flow of $\widehat{U}$. This theorem, indeed, consists 
in providing an explicit neighborhood of the manifold of collinear configurations such that orbits of the gradient flow of $\widehat{U}$ starting off from such a neighborhood become more and more collinear. In this section we will extend the validity of the $45^{\circ}$-Theorem to $s_{1}-\mathrm{CSBC}$ in $\mathbb{R}^{3}$. More precisely, we will show that there is a neighborhood of the manifold of collinear configurations along the $e_{1}$-axis such that orbits of the gradient flow of $\widehat{U}$ starting off from such a neighborhood become more and more collinear along the $e_{1}$-axis.

Such a $45^{\circ}$-Theorem for $s_{1}-$ CSBC holds for any $S=\operatorname{diag}\left(s_{1}, s_{2}, 1\right)$, and actually even for every $d \geq 3$. However, to keep the discussion as elementary as possible we assume hereafter that $S=\operatorname{diag}(s, 1,1)$ for some $s>1$, which is actually the case we will be interested in in Sect. 6. We set

$$
S_{M}:=\left[\begin{array}{lll}
m_{1} S & & \\
& \ddots & \\
& & m_{n} S
\end{array}\right]
$$

The gradient flow equation of $\widehat{U}$ is given by

$$
\dot{q}=S_{M}^{-1} \nabla U(q)+U(q) q=\widetilde{\nabla} \widehat{U}(q)
$$

Definition 5.1 The COLLINEARITY ANGLE of the configuration vector $q \in \widehat{\mathbb{S}}$ w.r.t. the $e_{1}$ direction is

$$
\vartheta(q):=\max _{i \neq j} \varangle\left(q_{i}-q_{j}, e_{1}\right) .
$$

Here $\varangle(v, w)$ denotes the angle between the two vectors $v, w \in \mathbb{R}^{3}$.

Theorem 5.2 ( $45^{\circ}$-Theorem for $\left.s_{1}-\mathrm{CSBC}\right)$. Consider the S-balanced configuration problem (2.1) on $\mathbb{R}^{3}$ with

$$
S=\operatorname{diag}(s, 1,1), \quad s>1 .
$$

Then the set

$$
\left\{q \in \widehat{S} \mid q \text { is collinear along the } e_{1} \text {-axis }\right\}
$$

is an attractor for the gradient flow of $\widehat{U}$. More precisely, the function pointwise defined in Eq. (5.2) is a Lyapunov function on

$$
\mathscr{V}:=\left\{q \mid \vartheta(q) \in\left(0,45^{\circ}\right]\right\}
$$

for $\widetilde{\nabla} \widehat{U}$. In particular there are no non-collinear $\operatorname{SBC}$ for $\vartheta(q(t)) \in\left(0,45^{\circ}\right]$.

Remark 5.3 A word by word generalization of Moeckel's $45^{\circ}$-Theorem (stating that the set of all configurations which are collinear along some line is an attractor for the gradient flow of $\widehat{U}$ ) cannot hold for SBC, as CSBC can be local minima of $\widehat{U}$ for suitable choices of $s>1$. In fact, a priori there is not even a reason why the set of collinear configurations should be invariant under the gradient flow.

Remark 5.4 $S$-balanced configuration in $\mathbb{R}^{3}$ with $S=\operatorname{diag}(s, 1,1)$ are of great interest since they produce relative equilibria of the $n$-body problem in $\mathbb{R}^{4}$ via "complexification". Notice that, unlike the case considered in Sects.2-4 (where all eigenvalues of $S$ had multiplicity one), here there is no need to complexify the plane $\{0\} \times \mathbb{R}^{2}$, but rather we just have to complexify the (real) line corresponding to the eigenvalue $s$. This fact can be equivalently explained as follows: if we consider the $S$-balanced configurations problem in $\mathbb{R}^{4}$ with matrix 
$S=\operatorname{diag}(s, s, 1,1)$, then restricting our attention only to $\mathrm{SBC}$ contained in $\{0\} \times \mathbb{R}^{3}$ would yield precisely the $S$-balanced problem in $\mathbb{R}^{3}$ with matrix $S=\operatorname{diag}(s, 1,1)$ considered above.

Proof We set $q=(x, y, z)^{\top}$. Choose $(i, j)$ such that

$$
\vartheta(q)=\varangle\left(q_{i}-q_{j}, e_{1}\right), \quad x_{i}-x_{j}>0 .
$$

By construction, all other $q_{k}$ must lie in the intersection of the two cones of angle $\theta(q)$ with respect to $e_{1}$ at $q_{i}$ and $q_{j}$, which consists of the segment joining $q_{j}$ with $q_{i}$ and the outer half-cones. Since by assumption $\theta(q) \leq 45^{\circ}$, the two half-cones lie on opposite sides of the orthogonal bisector $B$ of $q_{j}$ and $q_{i}$, which we recall is the plane orthogonal to $q_{i}-q_{j}$ through the middle point of the segment joining $q_{j}$ to $q_{i}$. By a simple minimality argument we can suppose without loss of generality that none of the remaining $q_{k}$ lie in the segment joining $q_{j}$ to $q_{i}$.

Since for all $k$ we have

$$
\begin{aligned}
\dot{q}_{k} & =m_{k}^{-1} S^{-1} \nabla_{k} U(q)+U(q) q_{k} \\
& =m_{k}^{-1} \nabla_{k} U(q)+U(q) q_{k}-m_{k}^{-1}\left(1-s^{-1}\right) \cdot \operatorname{pr}_{1}\left(\nabla_{k} U(q)\right),
\end{aligned}
$$

where

$$
\operatorname{pr}_{1}\left(\nabla_{k} U(q)\right)=\left(\sum_{\ell \neq k} m_{\ell} m_{k} \cdot \frac{x_{\ell}-x_{k}}{r_{\ell k}^{3}}\right) \cdot e_{1}
$$

is the projection of $\nabla_{k} U(q)$ onto the $x$-axis, we get that

$$
\begin{aligned}
\dot{q}_{i}-\dot{q}_{j} & =m_{i}^{-1} \nabla_{i} U(q)-m_{j}^{-1} \nabla_{j} U(q) \\
& -\left(1-s^{-1}\right)\left[m_{i}^{-1} \cdot \operatorname{pr}_{1}\left(\nabla_{i} U(q)\right)-m_{j}^{-1} \cdot \operatorname{pr}_{1}\left(\nabla_{j} U(q)\right)\right]+U(q)\left(q_{i}-q_{j}\right) .
\end{aligned}
$$

Set $\alpha(t):=\varangle\left(q_{i}(t)-q_{j}(t), e_{1}\right)$. By the assumption $x_{i}-x_{j}>0$, we have

$$
\cos \alpha(t)=\left\langle\frac{q_{i}(t)-q_{j}(t)}{r_{i j}(t)}, e_{1}\right\rangle>0
$$

Differentiating w.r.t. $t$ yields (the dependence on $t$ is hereafter dropped for notational convenience)

$$
\begin{aligned}
-\dot{\alpha} \sin \alpha & =\left\langle\frac{\dot{q}_{i}-\dot{q}_{j}}{r_{i j}}, e_{1}\right\rangle-\left\langle\frac{\left\langle\left(q_{i}-q_{j}\right), e_{1}\right\rangle}{r_{i j}^{3}}\left(q_{i}-q_{j}\right),\left(\dot{q}_{i}-\dot{q}_{j}\right)\right\rangle \\
& =\left\langle\frac{\dot{q}_{i}-\dot{q}_{j}}{r_{i j}},\left[e_{1}-\frac{\left\langle\left(q_{i}-q_{j}\right), e_{1}\right\rangle}{r_{i j}^{2}}\left(q_{i}-q_{j}\right)\right]\right\rangle \\
& =\left\langle\frac{\dot{q}_{i}-\dot{q}_{j}}{r_{i j}}, e_{1}^{B}\right\rangle
\end{aligned}
$$

where $e_{1}^{B}$ denotes the orthogonal projection of $e_{1}$ on the orthogonal bisector $B$ of $q_{i}$ and $q_{j}$. Observe that

$$
\left\langle e_{1}, e_{1}^{B}\right\rangle>0 .
$$


By using Eqs. (5.3) and (5.4) and by taking into account that $\left\langle q_{i}-q_{j}, e_{1}^{B}\right\rangle=0$, we get that

$$
\begin{aligned}
-\dot{\alpha} \sin \alpha= & \left\langle\frac{1}{r_{i j}}\left[m_{i}^{-1} \nabla_{i} U(q)-m_{j}^{-1} \nabla_{j} U(q)\right], e_{1}^{B}\right\rangle \\
& -\frac{1-s^{-1}}{r_{i j}}\left\langle m_{i}^{-1} \cdot \operatorname{pr}_{1}\left(\nabla_{i} U(q)\right)-m_{j}^{-1} \cdot \operatorname{pr}_{1}\left(\nabla_{j} U(q)\right), e_{1}^{B}\right\rangle \\
= & \frac{1}{r_{i j}}\left(\sum_{k \neq i} m_{k} \frac{q_{k}-q_{i}}{r_{i k}^{3}} e_{1}^{B}-\sum_{k \neq j} m_{k} \frac{q_{k}-q_{j}}{r_{j k}^{3}} e_{1}^{B}\right) \\
& +\frac{1-s^{-1}}{r_{i j}}\left(\sum_{k \neq j} m_{k} \frac{x_{k}-x_{j}}{r_{j k}^{3}}-\sum_{k \neq i} m_{k} \frac{x_{k}-x_{i}}{r_{i k}^{3}}\right)\left\langle e_{1}, e_{1}^{B}\right\rangle .
\end{aligned}
$$

By arguing precisely as in [10, P. 62-63], we get that that the first summand in the (RHS) of Eq. (5.5) is non-negative (actually, positive provided $q$ is not collinear along the line determined by $q_{j}$ and $q_{i}$ ). The last summand in the RHS of Eq. (5.5) is the product of the positive number $\left(1-s^{-1}\right) r_{i j}^{-1} \cdot\left\langle e_{1}, e_{1}^{B}\right\rangle$ with

$$
\sum_{k \neq j, i} m_{k}\left(\frac{x_{k}-x_{j}}{r_{j k}^{3}}-\frac{x_{k}-x_{i}}{r_{i k}^{3}}\right)+m_{i} \frac{x_{i}-x_{j}}{r_{j i}^{3}}-m_{j} \frac{x_{j}-x_{i}}{r_{i j}^{3}} .
$$

The last two terms in Eq. (5.6) are positive by the assumption $x_{i}-x_{j}>0$. Moreover, since by assumption none of the $q_{k}$ lies in the segment joining $q_{j}$ with $q_{i}$, for every $k \neq i, j$ we either have $x_{k}>x_{i}>x_{j}$ or $x_{k}<x_{j}<x_{i}$. If the former case holds, then $q_{k}$ lies in the outer cone at $q_{i}$ and hence the quantities

$$
\frac{x_{k}-x_{j}}{r_{j k}^{3}} \text { and } \frac{x_{k}-x_{i}}{r_{i k}^{3}}
$$

are both positive and the first one is larger than the second one. If the latter case holds, then $q_{k}$ lies in the outer cone at $q_{j}$ and the quantities

$$
\frac{x_{k}-x_{j}}{r_{j k}^{3}} \text { and } \frac{x_{k}-x_{i}}{r_{i k}^{3}}
$$

are both negative and the second one is in absolute value larger than the first one. Overall, this shows that the expression in Eq. (5.6) (thus also the RHS of Eq. (5.5)) is always positive. Therefore, by (5.5) we can infer that $-\dot{\alpha} \sin \alpha>0$, thus completing the proof.

A direct consequence of the $45^{\circ}$-theorem for $s_{1}-\mathrm{CSBC}$ is the following.

Corollary 5.5 The set

$$
\mathbb{Y}:=\left\{q=\left[\left(\begin{array}{c}
x_{1} \\
0 \\
0
\end{array}\right), \ldots,\left(\begin{array}{c}
x_{n} \\
0 \\
0
\end{array}\right)\right] \in \mathbb{R}^{3 n} \mid \sum_{i=1}^{n} m_{i} x_{i}=0,\|q\|_{S_{M}}^{2}=\frac{1}{s}\right\}
$$

is an ATTRACTOR for the gradient flow of $\widehat{U}$.

The set $\mathscr{V}$ is invariant under the gradient flow and the function $t \mapsto \vartheta(q(t))$ is strictly decreasing along gradient flow lines, provided $\vartheta(q(0)) \in\left(0,45^{\circ}\right]$. 
Remark 5.6 Corollary 5.5 allows us to remove the set $\mathbb{Y}$ from $\widehat{\mathbb{S}}$ before quotienting out by the (diagonal) $\mathrm{SO}(2)$-action on $\widehat{\mathbb{S}}$ induced by rotations in the $\widehat{y O z}$-plane, which we recall is the symmetry group of Eq. (2.1) in case $S=\operatorname{diag}(s, 1,1)$. Since $\mathbb{Y}$ is precisely the singular set of the $\mathrm{SO}(2)$-action, we obtain that

$$
\overline{\mathcal{S}}:=(\widehat{\mathbb{S}} \backslash \mathbb{Y}) / \operatorname{SO}(2)
$$

is a manifold. Such an argument is actually very similar to the one used by Merkel in [8] in the case of spatial CC (the main difference being that we remove only collinear configuration in the $e_{1}$-direction instead of the whole manifold of collinear configurations), unlike in Merkel's case however the lower bound that we will obtain is much larger than McCord's lower bound on the number of planar CC (cfr. Sect. 6).

The goal of the next section will be to compute the Poincaré polynomial of $\overline{\mathcal{S}}$. This will give us - via the "complexification argument" discussed in Remark 5.4 - a lower bound on the number of SBC in $\mathbb{R}^{4}$ with matrix diag $(s, s, 1,1)$, that will be then compared with the lower bounds provided by Theorems 4.2 and 4.5 via the reduction to (H1) argument.

We finish this section stating the general $45^{\circ}$-theorem for $s_{1}-\mathrm{CSBC}$. The proof can be obtained from the one of Theorem 5.2 with some minor modifications and will be omitted.

Theorem 5.7 ( $45^{\circ}$-theorem for $s_{1}$-collinear SBC). For $d \geq 2$, let $s_{1}>s_{2}>\ldots>s_{d-1}>$ $s_{d}=1$ be positive real numbers and $\mu_{1}, \ldots, \mu_{d} \in \mathbb{N}$ be natural numbers. Consider the S-balanced configurations problem (2.1) on $\mathbb{R}^{D}, D:=\mu_{1}+\ldots+\mu_{d} \geq d$, with

$$
S=\operatorname{diag}(\underbrace{s_{1}, \ldots, s_{1}}_{\mu_{1} \text {-times }}, \ldots, \underbrace{s_{d-1}, \ldots, s_{d-1}}_{\mu_{d-1} \text {-times }}, \underbrace{1, \ldots, 1}_{\mu_{d} \text {-times }}) .
$$

Then, the set

$$
\left\{q \in \widehat{\mathbb{S}} \mid q \text { is collinear along some line in } \mathbb{R}^{\mu_{1}} \times\{0\} \subset \mathbb{R}^{D}\right\}
$$

is an attractor for the gradient flow of $\widehat{U}$. More precisely, the COLLINEARITY FUNCTION

$$
\theta(q):=\min _{L} \max _{i \neq j} \varangle\left(q_{i}-q_{j}, L\right),
$$

where $L$ runs all over the lines through the origin in $\mathbb{R}^{\mu_{1}} \times(0)$, is a Lyapounov function for the gradient vector field of $\widehat{U}$ on the set $\left\{q \mid \theta(q) \in\left(0,45^{\circ}\right]\right\}$.

Remark 5.8 The singular set of the action of the symmetry group of Eq. 2.1 does not consist of only collinear configurations as soon as $d \geq 4$. This implies that the quotient of $\widehat{\mathbb{S}}$ by the group action will not be a manifold even if the $45^{\circ}$-theorem is available in any dimension. Indeed, the whole singular set cannot be removed, in general. This is already the case for planar CC, which are in general not an attractor for the gradient flow. Besides that, the computations of the homology of the (singular) quotient are unaccessible for large values of $d$, and even if in the particular case considered in this section and in Section 6, we succeed to obtain a quotient manifold and to compute (though with some effort) its homology, in the count of SBC we still have to keep in mind that planar SBC which are contained in the $\widehat{y O z}$-plane are actually $\mathrm{CC}$, whereas the non-collinear SBC provided by Theorems 4.2 and 4.5 cannot be CC.

All these facts put on evidence, once again, the importance of the reduction to (H1) argument. 


\section{A Lower Bound on the Number of SBC in $\mathbb{R}^{4}$ á la McCord}

In this last section we compute the homology of the manifold $\overline{\mathcal{S}}$ defined in (5.7) and, by means of this information, give lower bounds on the number of SBC in $\mathbb{R}^{4}$ with matrix $\operatorname{diag}(s, s, 1,1)$ that will be then compared with the ones obtained in Theorem 4.2.

Theorem 6.1 For masses $m_{1}, \ldots, m_{n}>0$ consider the $S$-balanced configurations problem (2.1) on $\mathbb{R}^{3}$ with $S=\operatorname{diag}(s, 1,1), s>1$. Under Assumption (H2), there exist at least

$$
n !\left(\sum_{j=2}^{n-1} \frac{1}{j}+\frac{2}{n}\right)
$$

$S O(2)$-families $^{2}$ of SBC. As a corollary, for the corresponding S-balanced configurations problem (2.1) on $\mathbb{R}^{4}$ with $S=\operatorname{diag}(s, s, 1,1)$ there are at least the same number as above of $S O(2) \times S O(2)$-families of $\mathrm{SBC}$.

Remark 6.2 The lower bound provided by Theorem 6.1 is much bigger than the ones in Theorems 4.2 and 4.5, as it asymptotically grows like $n ! \log n$. Moreover, unlike Theorems 4.2 and 4.5 , such a lower bound is independent of any spectral gap condition for $s$. The drawback is that we cannot a priori exclude that all SBC we find are in fact CC contained in $\{0\} \times \mathbb{R}^{2}$. However, Theorem 6.1 would be interesting even if this were the case, as the estimate provided by (6.1) is larger than McCord's estimate [7] by a factor 2 .

The proof of Theorem 6.1 will take up the rest of the section. The two major ingredients are:

1. Corollary 5.5: The manifold $\mathbb{Y}$ is an attractor for the gradient flow of $\widehat{U}$, and the neighborhood $\mathscr{V}$ of $\mathbb{Y}$ does not contain SBC other than the $s_{1}$-collinear SBC.

2. Shub's lemma for $\mathrm{SBC}^{3}$ : $\mathrm{SBC}$ cannot accumulate on the singular set $\Delta$. In particular, there exists an invariant neighborhood of $\Delta$ containing no SBC.

We set $\widehat{\mathbb{Y}}:=\mathbb{Y} \backslash \Delta$ and assume hereafter that $n \geq 4$, as for $n=3$ some arguments need some slight modification. We start by collecting some well-known facts. The first two are direct consequence of the fact that both $\mathbb{S}$ and $\mathbb{Y}$ are homological spheres:

$$
H_{*}(\mathbb{S})=\left\{\begin{array}{c}
\mathbb{R} \text { if } *=0,3 n-4, \\
0 \quad \text { otherwise, }
\end{array} \quad H_{*}(\mathbb{Y})=\left\{\begin{array}{c}
\mathbb{R} \text { if } *=0, n-2, \\
0 \quad \text { otherwise }
\end{array}\right.\right.
$$

It is also readily seen that $\Delta$ is a codimensional-3-submanifold of $\mathbb{S}$. In particular, the set

$$
\mathbb{S} \backslash(\Delta \cup \mathbb{Y})
$$

is simply connected. The set $\widehat{\mathbb{Y}}$ consists of $n$ ! connected components (corresponding to the orderings of $n$ distinct points on the line), each of which is topologically a $(n-2)$-dimensional disk. In particular

$$
H_{*}(\widehat{\mathbb{Y}})=\left\{\begin{array}{cc}
\mathbb{R}^{n !} & \text { if } *=0 \\
0 & \text { otherwise }
\end{array}\right.
$$

\footnotetext{
2 As the problem is $S O(2)$-invariant, SBC always come in $S O(2)$-families.

3 The proof of the original Shub's lemma carries over word by word to SBC.
} 
By the universal coefficients theorem and Lemma 3.1 we also have

$$
\widetilde{H}_{*}(\widehat{\mathbb{S}}) \cong \widetilde{H}^{*}(\widehat{\mathbb{S}})= \begin{cases}\mathbb{R}^{c_{j}} & \text { if } *=2 j, \\ 0 & \text { otherwise }\end{cases}
$$

where $c_{j}$ is the $j$-th coefficient of the polynomial $p(t)=(1+t) \cdot \ldots \cdot(1+(n-1) t)$ and $\widetilde{H}_{*}\left(\right.$ resp. $\left.\widetilde{H}^{*}\right)$ denotes the reduced homology (resp. cohomology). Therefore, by Alexander duality,

$$
\widetilde{H}_{*}(\Delta) \cong \widetilde{H}^{3 n-5-*}(\widehat{\mathbb{S}})=\left\{\begin{array}{cr}
\mathbb{R}^{c_{j}} & \text { if } *=3 n-5-2 j, \\
0 & \text { otherwise }
\end{array}\right.
$$

In particular, $H_{*}(\Delta)$ vanishes in all degrees smaller than $n-4$ (except $\left.*=0\right)$, and

$$
\begin{aligned}
H_{n-3}(\Delta) & \cong \mathbb{R}^{c_{n-1}}=\mathbb{R}^{(n-1) !}, \\
H_{n-1}(\Delta) & \cong \mathbb{R}^{c_{n-2}}=\mathbb{R}^{(n-1) ! \sum_{j=1}^{n-1} \frac{1}{j}}, \\
\cdots & \\
H_{3 n-7}(\Delta) & \cong \mathbb{R}^{c_{1}}=\mathbb{R}^{\frac{n(n+1)}{2}} .
\end{aligned}
$$

\subsection{Homology of Some Intermediate Manifolds}

The computation of the homology of $\overline{\mathcal{S}}=(\mathbb{S} \backslash(\Delta \cup \mathbb{Y})) / \mathrm{SO}(2)$ is divided in the following intermediate steps:

(Step 1) We compute the homology of $\mathbb{Y} \cup \Delta$.

(Step 2) We compute the reduced homology of $\mathbb{S} \backslash(\mathbb{Y} \cup \Delta$ ) using Alexander duality.

(Step 3) We conclude using the Gysin long exact sequence of the fibration

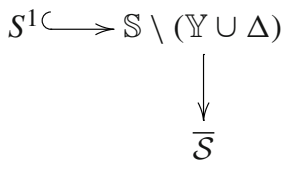

In this subsection we will discuss Steps 1 and 2, leaving Step 3 for the next subsection. Thus, we start computing the homology of $\mathbb{Y} \cup \Delta$ by using the Mayer-Vietoris sequence and the homological properties of $\mathbb{Y}, \mathbb{Y} \cap \Delta$, and $\Delta$. Since

$$
\mathbb{Y}=(\mathbb{Y} \backslash \Delta) \cup(\mathbb{Y} \cap \Delta)=\widehat{\mathbb{Y}} \cup(\mathbb{Y} \cap \Delta),
$$

denoting by $\widehat{\Delta}$ a fattened open neighborhood of $\Delta$ in $\mathbb{Y}$, we get that

$$
\mathbb{Y}=\widehat{\mathbb{Y}} \cup(\mathbb{Y} \cap \widehat{\Delta})
$$

Each connected component of the intersection $\widehat{\mathbb{Y}} \cap(\mathbb{Y} \cap \widehat{\Delta})$ is homotopy equivalent to $S^{n-3}$ (recall that the connected components of $\widehat{\mathbb{Y}}$ are homotopically $(n-2)$-dimensional disks). Since we have $n$ ! connected components in total, we obtain

$$
H_{*}\left(\widehat { \mathbb { Y } } \cap ( \mathbb { Y } \cap \widehat { \Delta } ) \cong \left\{\begin{array}{rr}
\mathbb{R}^{n !} & \text { if } *=0, n-3 \\
0 & \text { otherwise. }
\end{array}\right.\right.
$$

To compute the homology of $\mathbb{Y} \cap \Delta$, we start observing that $\Delta$ is an (ANR) in $\mathbb{Y}$ and hence

$$
H_{*}(\mathbb{Y} \cap \Delta) \cong H_{*}(\mathbb{Y} \cap \widehat{\Delta}) .
$$


By using Mayer-Vietoris we obtain the following exact sequence

$$
\cdots \longrightarrow H_{k+1}(\mathbb{Y}) \longrightarrow H_{k}(\widehat{\mathbb{Y}} \cap(\mathbb{Y} \cap \widehat{\Delta})) \longrightarrow H_{k}(\widehat{\mathbb{Y}}) \oplus H_{k}(\mathbb{Y} \cap \widehat{\Delta}) \longrightarrow H_{k}(\mathbb{Y}) \longrightarrow \cdots
$$

In particular, using all available information for $k=0$ we obtain

$$
\cdots \longrightarrow 0 \longrightarrow \mathbb{R}^{n !} \longrightarrow \mathbb{R}^{n !} \oplus H_{0}(\mathbb{Y} \cap \widehat{\Delta}) \longrightarrow \mathbb{R} \longrightarrow 0
$$

which yields $H_{0}(\mathbb{Y} \cap \widehat{\Delta}) \cong \mathbb{R}$, whereas the long exact sequence for $k=1, \ldots, n-3$ implies

$$
H_{j}(\mathbb{Y} \cap \widehat{\Delta}) \cong 0, \quad \forall j=1, \ldots, n-4 .
$$

Finally, the long exact sequence for $k=n-2$ reads

$$
\cdots \longrightarrow 0 \longrightarrow 0 \oplus H_{n-2}(\mathbb{Y} \cap \widehat{\Delta}) \longrightarrow \mathbb{R} \longrightarrow \mathbb{R}^{n !} \longrightarrow 0 \oplus H_{n-3}(\mathbb{Y} \cap \widehat{\Delta}) \longrightarrow 0 \longrightarrow \cdots
$$

Since $\mathbb{Y} \cap \widehat{\Delta}$ is homotopy equivalent to $\mathbb{Y} \cap \Delta$, which is a codimension 1 subset of $\mathbb{Y}$, we have in particular that $H_{n-2}(\mathbb{Y} \cap \widehat{\Delta})=0$. Hence, the sequence above becomes

$$
0 \longrightarrow \mathbb{R} \longrightarrow \mathbb{R}^{n !} \longrightarrow 0 \oplus H_{n-3}(\mathbb{Y} \cap \widehat{\Delta}) \longrightarrow 0
$$

By exactness we conclude that

$$
H_{n-3}(\mathbb{Y} \cap \widehat{\Delta}) \cong \mathbb{R}^{n !-1} .
$$

Summing up, we have proved that

$$
H_{*}(\mathbb{Y} \cap \Delta) \cong H_{*}(\mathbb{Y} \cap \widehat{\Delta})=\left\{\begin{array}{cr}
\mathbb{R} & \text { if } *=0, \\
\mathbb{R}^{n !-1} & \text { if } *=n-3, \\
0 & \text { otherwise. }
\end{array}\right.
$$

We are now ready to compute the homology of $\mathbb{Y} \cup \Delta$. To do this we use the Mayer-Vietoris sequence associated with $\mathbb{Y}, \mathbb{Y} \cap \Delta$ and $\Delta$ :

$$
\cdots \longrightarrow H_{k}(\mathbb{Y} \cap \Delta) \longrightarrow H_{k}(\mathbb{Y}) \oplus H_{k}(\Delta) \longrightarrow H_{k}(\mathbb{Y} \cup \Delta) \longrightarrow H_{k-1}(\mathbb{Y} \cap \Delta) \longrightarrow \cdots
$$

We immediately see that the long exact sequence above implies

$$
H_{*}(\mathbb{Y} \cup \Delta)=\{0\}, \quad \text { for } *=1, \ldots, n-4,
$$

whereas for $*=n-1, \ldots, 3 n-7$ we have

$$
H_{*}(\mathbb{Y} \cup \Delta) \cong H_{*}(\Delta) .
$$

For $k=n-2$ we get

$$
0 \longrightarrow \mathbb{R} \longrightarrow H_{n-2}(\mathbb{Y} \cup \Delta) \longrightarrow \mathbb{R}^{n !-1} \longrightarrow{ }^{g} \longrightarrow \mathbb{R}^{(n-1) !} \stackrel{h}{\longrightarrow} H_{n-3}(\mathbb{Y} \cup \Delta) \longrightarrow 0
$$

Since $\mathbb{Y} \cup \Delta$ is homotopy equivalent to the union of $\Delta$ together with $n$ ! disks of dimension $(n-2)$, we get

$$
H_{n-3}(\mathbb{Y} \cup \Delta) \cong H_{n-3}(\Delta) \cong \mathbb{R}^{(n-1) !}
$$

and hence in particular $h$ is an isomorphism. By exactness, this implies that $\operatorname{Im} g=\{0\}$. Therefore, the exact sequence can be rewritten as

$$
0 \longrightarrow \mathbb{R} \longrightarrow H_{n-2}(\mathbb{Y} \cup \Delta) \longrightarrow \mathbb{R}^{n !-1} \longrightarrow 0
$$


and this readily implies that

$$
H_{n-2}(\mathbb{Y} \cup \Delta) \cong \mathbb{R}^{n !} .
$$

Summarizing, we have proved that

$$
H_{*}(\mathbb{Y} \cup \Delta) \cong\left\{\begin{array}{cr}
\mathbb{R} & \text { if } *=0, \\
\mathbb{R}^{n !} & \text { if } *=n-2, \\
\mathbb{R}^{c_{j}} \quad \text { if } *=3 n-5-2 j, j=1, \ldots, n-1, & \text { otherwise. }
\end{array}\right.
$$

Using Alexander duality and the universal coefficients theorem, we get

$$
\widetilde{H}_{*}(\mathbb{S} \backslash(\mathbb{Y} \cup \Delta)) \cong \widetilde{H}^{3 n-5-*}(\mathbb{S} \backslash(\mathbb{Y} \cup \Delta)) \cong \widetilde{H}_{*}(\mathbb{Y} \cup \Delta)
$$

and hence finally

$$
\left.H_{*}(\mathbb{S} \backslash \mathbb{Y} \cup \Delta)\right) \cong\left\{\begin{array}{cr}
\mathbb{R} & \text { if } *=0, \\
\mathbb{R}^{n !} & \text { if } *=2 n-3, \\
\mathbb{R}^{c_{j}} \quad \text { if } *=2 j, j=1, \ldots, n-1, & \text { otherwise. }
\end{array}\right.
$$

\subsection{The Homology of $\overline{\mathcal{S}}$}

Let us now consider the fibration

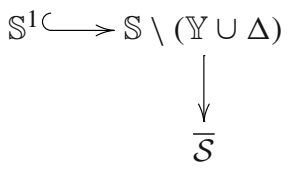

Since $\mathbb{S} \backslash(\mathbb{Y} \cup \Delta))$ is simply connected, the exact sequence of homotopy groups

$$
\cdots \longrightarrow \pi_{1}(\mathbb{S} \backslash(\mathbb{Y} \cup \Delta)) \longrightarrow \pi_{1}(\overline{\mathcal{S}}) \longrightarrow \pi_{0}\left(\mathbb{S}^{1}\right)
$$

yields that $\pi_{1}(\overline{\mathcal{S}})=\{0\}$. Therefore, the fibration is homologically orientable. Using the information provided in Sect. 6.1, we can rewrite the Gysin long exact sequence of the fibration

$$
\cdots \longrightarrow H_{k+1}\left(\mathbb{S} \backslash(\mathbb{Y} \cup \Delta) \longrightarrow H_{k+1}(\overline{\mathcal{S}}) \longrightarrow H_{k-1}(\overline{\mathcal{S}}) \longrightarrow H_{k}(\mathbb{S} \backslash(\mathbb{Y} \cup \Delta) \longrightarrow \cdots\right.
$$


as follows:

$$
\begin{aligned}
& \ldots \longrightarrow H_{2 n-3}(\overline{\mathcal{S}}) \longrightarrow \underbrace{H_{2 n-2}(\mathbb{S} \backslash(\mathbb{Y} \cup \Delta)}_{=0} \longrightarrow H_{2 n-2}(\overline{\mathcal{S}}) \longrightarrow \\
& H_{2 n-4}(\overline{\mathcal{S}}) \longrightarrow \underbrace{H_{2 n-3}(\mathbb{S} \backslash(\mathbb{Y} \cup \Delta)}_{=\mathbb{R}^{n !}} \longrightarrow H_{2 n-3}(\overline{\mathcal{S}}) \longrightarrow H_{2 n-5}(\overline{\mathcal{S}}) \longrightarrow \\
& \underbrace{H_{2 n-4}(\mathbb{S} \backslash(\mathbb{Y} \cup \Delta)}_{=\mathbb{R}^{c_{n-2}}} \longrightarrow H_{2 n-4}(\overline{\mathcal{S}}) \longrightarrow \underbrace{H_{2 n-5}(\mathbb{S} \backslash(\mathbb{Y} \cup \Delta)}_{=0} \rightarrow \\
& H_{2}(\overline{\mathcal{S}}) \longrightarrow H_{0}(\overline{\mathcal{S}}) \longrightarrow \underbrace{H_{1}(\mathbb{S} \backslash(\mathbb{Y} \cup \Delta)}_{=0} \longrightarrow \underbrace{H_{1}(\overline{\mathcal{S}})}_{=0} \longrightarrow \\
& 0 \longrightarrow \underbrace{H_{0}(\mathbb{S} \backslash(\mathbb{Y} \cup \Delta)}_{=0} \longrightarrow H_{0}(\overline{\mathcal{S}}) \longrightarrow 0
\end{aligned}
$$

Therefore, $H_{0}(\overline{\mathcal{S}}) \cong \mathbb{R}$ and $H_{1}(\overline{\mathcal{S}}) \cong\{0\}$. Reading the long exact sequence backwards we obtain

$$
0=H_{1}(\overline{\mathcal{S}}) \longrightarrow \underbrace{H_{2}(\mathbb{S} \backslash(\mathbb{Y} \cup \Delta)}_{=\mathbb{R}^{c_{1}}} \longrightarrow H_{2}(\overline{\mathcal{S}}) \longrightarrow \mathbb{R} \longrightarrow 0
$$

which implies $H_{2}(\overline{\mathcal{S}}) \cong \mathbb{R} \oplus \mathbb{R}^{c_{1}}$. Similarly,

$$
0=H_{3}(\mathbb{S} \backslash(\mathbb{Y} \cup \Delta)) \longrightarrow H_{3}(\overline{\mathcal{S}}) \longrightarrow \underbrace{H_{1}(\overline{\mathcal{S}})}_{=0} \longrightarrow \ldots
$$

implies $H_{3}(\overline{\mathcal{S}})=0$. Computing further yields

$$
0=H_{3}(\overline{\mathcal{S}}) \longrightarrow H_{4}(\mathbb{S} \backslash(\mathbb{Y} \cup \Delta)) \longrightarrow H_{4}(\overline{\mathcal{S}}) \longrightarrow H_{2}(\overline{\mathcal{S}}) \longrightarrow 0
$$

implying that

$$
H_{4}(\overline{\mathcal{S}}) \cong H_{2}(\overline{\mathcal{S}}) \oplus H_{4}(\mathbb{S} \backslash(\mathbb{Y} \cup \Delta)) \cong \mathbb{R} \oplus \mathbb{R}^{c_{1}} \oplus \mathbb{R}^{c_{2}}
$$

and arguing recursively we obtain for all but the top degree terms the following

Lemma 6.3 For every $k=0, \ldots, n-3$ we have:

$$
\begin{aligned}
H_{2 k+1}(\overline{\mathcal{S}}) & =\{0\}, \\
H_{2 k}(\overline{\mathcal{S}}) & =H_{0}(\overline{\mathcal{S}}) \oplus \bigoplus_{j=1}^{k} H_{2 j}(\mathbb{S} \backslash(\mathbb{Y} \cup \Delta))=\mathbb{R}^{\sum_{j=0}^{k} c_{j}} .
\end{aligned}
$$


For the top degree terms we first observe that

$$
0=H_{2 n-5}(\overline{\mathcal{S}}) \longrightarrow \underbrace{H_{2 n-4}(\mathbb{S} \backslash(\mathbb{Y} \cup \Delta))}_{=\mathbb{R}^{c_{n-2}}} \longrightarrow H_{2 n-4}(\overline{\mathcal{S}}) \longrightarrow H_{2 n-6}(\overline{\mathcal{S}}) \longrightarrow 0
$$

implies that

$$
H_{2 n-4}(\overline{\mathcal{S}}) \cong \mathbb{R}^{\sum_{j=0}^{n-2} c_{j}} .
$$

Moreover, arguing recursively we deduce from

$$
\begin{gathered}
\underbrace{H_{2 n}(\mathbb{S} \backslash(\mathbb{Y} \cup \Delta))}_{=0} \longrightarrow H_{2 n}(\overline{\mathcal{S}}) \longrightarrow H_{2 n-2}(\overline{\mathcal{S}}) \longrightarrow \underbrace{H_{2 n-1}(\mathbb{S} \backslash(\mathbb{Y} \cup \Delta))}_{=0}, \\
\underbrace{H_{2 n+1}(\mathbb{S} \backslash(\mathbb{Y} \cup \Delta)}_{=0}) \longrightarrow H_{2 n+1}(\overline{\mathcal{S}}) \longrightarrow H_{2 n-1}(\overline{\mathcal{S}}) \longrightarrow \underbrace{H_{2 n}(\mathbb{S} \backslash(\mathbb{Y} \cup \Delta))}_{=0}
\end{gathered}
$$

and the fact that $\overline{\mathcal{S}}$ is finite dimensional that

$$
H_{j}(\overline{\mathcal{S}}) \cong 0, \quad \forall j \geq 2 n-2,
$$

as all such homology groups are isomorphic. Finally, from

$$
0 \longrightarrow H_{2 n-3}(\overline{\mathcal{S}}) \longrightarrow H_{2 n-2}(\mathbb{S} \backslash(\mathbb{Y} \cup \Delta)) \longrightarrow H_{2 n-2}(\overline{\mathcal{S}})=0
$$

we obtain using Part 1 in Proposition 3.2

$$
H_{2 n-3}(\overline{\mathcal{S}}) \cong \mathbb{R}^{n !-\sum_{j=0}^{n-2} c_{j}}=\mathbb{R}^{c_{n-1}}=\mathbb{R}^{(n-1) !}
$$

Summarizing all the previous computations, we finally get the following result.

Theorem 6.4 The homology of $\overline{\mathcal{S}}$ is given by

$$
H_{*}(\overline{\mathcal{S}}) \cong\left\{\begin{array}{cr}
\mathbb{R}^{\sum_{j=0}^{k} c_{j}} \text { if } *=2 k, & k=0, \ldots, n-2 \\
\mathbb{R}^{(n-1) !} & \text { if } *=2 n-3 \\
0 & \text { otherwise }
\end{array}\right.
$$

A new lower bound on the number of critical points of $\widehat{U}$ assuming non-degeneracy is given now by the sum of the Betti numbers of $\overline{\mathcal{S}}$ :

$$
(n-1) !+\sum_{k=0}^{n-2} \sum_{j=0}^{k} c_{j}=(n-1) !+\sum_{j=0}^{n-2} c_{j}(n-1-j) .
$$

The next lemma, which appears in [7] without proof, will be useful to find a closed form for (6.3).

Lemma 6.5 Denote by $\xi_{j}^{(n)}$ the jth coefficient of the polynomial

$$
(1+2 t) \cdot \ldots \cdot(1+(n-1) t) .
$$


Then,

$$
\sum_{j=0}^{n-2} \xi_{j}^{(n)}=\frac{n !}{2}, \quad \sum_{j=0}^{n-3} \xi_{j}^{(n)}(n-2-j)=\frac{n !}{2} h(n),
$$

where $h(n):=\sum_{j=3}^{n} \frac{1}{j}$.

Proof The first identity follows evaluating the polynomial at $t=1$. We now prove the second identity by induction over $n$. A straightforward computation shows that for $n=4$ both RHS and LHS are equal to 7. Suppose now that the claim be true for $n$. We want to show that

$$
\sum_{j=0}^{n-2} \xi_{j}^{(n+1)}(n-1-j)=\frac{(n+1) !}{2} h(n+1) .
$$

As one readily sees, we have

$$
\xi_{j}^{(n+1)}=\xi_{j}^{(n)}+n \xi_{j-1}^{(n)}, \quad \forall j=0, \ldots, n-1,
$$

where we set $\xi_{-1}^{(n)}=\xi_{n-1}^{(n)}:=0$. Therefore, the (LHS) of Eq. (6.4) can be rewritten by using the first identity and the inductive assumption as

$$
\begin{aligned}
\sum_{j=0}^{n-2} \xi_{j}^{(n+1)}(n-1-j) & =\sum_{j=0}^{n-2}\left(\xi_{j}^{(n)}+n \xi_{j-1}^{(n)}\right)(n-1-j) \\
& =\sum_{j=0}^{n-2} \xi_{j}^{(n)}+\sum_{j=0}^{n-3} \xi_{j}^{(n)}(n-2-j)+n \sum_{j=0}^{n-2} \xi_{j-1}^{(n)}(n-1-j) \\
& =\frac{n !}{2}+\frac{n !}{2} h(n)+n \sum_{j=0}^{n-3} \xi_{j}^{(n)}(n-2-j) \\
& =\frac{n !}{2}+\frac{n !}{2} h(n)+n \frac{n !}{2} h(n) \\
& =\frac{(n+1) !}{2} h(n+1) .
\end{aligned}
$$

This completes the proof.

For notational convenience, we hereafter drop the superscript from $\xi_{j}^{(n)}$. By the very definition of the coefficients $c_{j}$, we see that

$$
c_{j}=\xi_{j}+\xi_{j-1}, \quad \forall j=0, \ldots, n-1,
$$

where as above $\xi_{-1}=\xi_{n-1}:=0$. Therefore, using Lemma 6.5 we can rewrite (6.3) as

$$
\begin{aligned}
(n-1) !+\sum_{j=0}^{n-2} c_{j}(n-1-j) & =(n-1) !+\sum_{j=0}^{n-2}\left(\xi_{j}+\xi_{j-1}\right)(n-1-j) \\
& =(n-1) !+\sum_{j=0}^{n-2} \xi_{j}+\sum_{j=0}^{n-3} \xi_{j}(n-2-j)+\sum_{j=0}^{n-2} \xi_{j-1}(n-1-j)
\end{aligned}
$$




$$
\begin{aligned}
& =(n-1) !+\frac{n !}{2}+n ! h(n) \\
& =n !\left(h(n)+\frac{1}{2}+\frac{1}{n}\right) .
\end{aligned}
$$

This gives the desired lower bound on the number of critical points of $\widehat{U}$ on $\overline{\mathcal{S}}$ assuming nondegeneracy, thus completing the proof of Theorem 6.1. As already observed in Remark 6.2, such a set of critical points contains also $\mathrm{CC}$ in the $\widehat{y O z}$-plane, which are in virtue of [7] (taking into account also c) at least

$$
\frac{n !}{2}(h(n)+1)
$$

Acknowledgements We warmly thank the anonymous referee for his careful reading of the paper and for his precious comments and suggestions which enabled us to improve significantly the paper and remove several inaccuracies. Luca Asselle is partially supported by the DFG-grant 380257369 "Morse theoretical methods in Hamiltonian dynamics".

Funding Open Access funding enabled and organized by Projekt DEAL.

Open Access This article is licensed under a Creative Commons Attribution 4.0 International License, which permits use, sharing, adaptation, distribution and reproduction in any medium or format, as long as you give appropriate credit to the original author(s) and the source, provide a link to the Creative Commons licence, and indicate if changes were made. The images or other third party material in this article are included in the article's Creative Commons licence, unless indicated otherwise in a credit line to the material. If material is not included in the article's Creative Commons licence and your intended use is not permitted by statutory regulation or exceeds the permitted use, you will need to obtain permission directly from the copyright holder. To view a copy of this licence, visit http://creativecommons.org/licenses/by/4.0/.

\section{Appendix: A Proof of Lemma 3.8}

In this section we prove Lemma 3.8.

Lemma A.1 For every $n, j \in \mathbb{N}$ we have

$$
\int_{1}^{n} \frac{1}{i_{1}} \cdot \int_{i_{1}}^{n} \frac{1}{i_{2}} \cdot \ldots \cdot \int_{i_{j-1}}^{n} \frac{1}{i_{j}} \mathrm{~d} i_{j} \cdot \ldots \cdot \mathrm{d} i_{1}=\frac{1}{j !} \log ^{j}(n) .
$$

Proof We divide the proof in two steps:

Step 1. We prove by induction over $j \in \mathbb{N}$ that:

$$
\begin{aligned}
& \int_{1}^{n} \frac{1}{i_{1}} \cdot \int_{i_{1}}^{n} \frac{1}{i_{2}} \cdot \ldots \cdot \int_{i_{j-1}}^{n} \frac{1}{i_{j}} \mathrm{~d} i_{j} \cdot \ldots \cdot \mathrm{d} i_{1}=a_{j} \cdot \log ^{j}(n), \\
& \int_{i_{0}}^{n} \frac{1}{i_{1}} \cdot \int_{i_{1}}^{n} \frac{1}{i_{2}} \cdot \ldots \cdot \int_{i_{j-1}}^{n} \frac{1}{i_{j}} \mathrm{~d} i_{j} \cdot \ldots \cdot \mathrm{d} i_{1}=\sum_{k=0}^{j} \frac{(-1)^{k}}{k !} a_{j-k} \log ^{j-k}(n) \log ^{k}\left(i_{0}\right),
\end{aligned}
$$

where the $a_{j}$ 's satisfy the recursive relation

$$
\left\{\begin{array}{l}
a_{0}=a_{1}=1, \\
a_{j+1}=\sum_{k=0}^{j} \frac{(-1)^{k}}{(k+1) !} a_{j-k}, \quad \forall j \geq 1 .
\end{array}\right.
$$


The claim is trivial for $j=1$. Indeed

$$
\int_{1}^{n} \frac{1}{i_{1}} \mathrm{~d} i_{1}=\log (n)=a_{1} \cdot \log ^{1}(n)
$$

and

$$
\int_{i_{0}}^{n} \frac{1}{i_{1}} \mathrm{~d} i_{1}=\log (n)-\log \left(i_{0}\right)=\frac{(-1)^{0}}{0 !} a_{1-0} \log ^{1-0}(n)+\frac{(-1)^{1}}{1 !} a_{1-1} \log ^{1-1}(n) \log ^{1}\left(i_{0}\right) .
$$

Suppose now that the claim be true for $j$ and compute for $j+1$ using (A.2) for $j$ and the recursive relation (A.3) defining the $a_{j}$ 's:

$$
\begin{aligned}
\int_{1}^{n} \frac{1}{i_{1}} & \int_{i_{1}}^{n} \frac{1}{i_{2}} \cdot \ldots \cdot \int_{i_{j}}^{n} \frac{1}{i_{j+1}} \mathrm{~d} i_{j+1} \cdot \ldots \cdot \mathrm{d} i_{2} \mathrm{~d} i_{1} \\
& =\int_{1}^{n} \frac{1}{i_{1}} \cdot\left(\sum_{k=0}^{j} \frac{(-1)^{k}}{k !} a_{j-k} \log j-k(n) \log ^{k}\left(i_{1}\right)\right) \mathrm{d} i_{1} \\
& =\left[\sum_{k=0}^{j} \frac{(-1)^{k}}{(k+1) !} a_{j-k} \log ^{j-k}(n) \log ^{k+1}\left(i_{1}\right)\right]_{1}^{n} \\
& =\log ^{j+1}(n) \cdot \sum_{k=0}^{j} \frac{(-1)^{k}}{(k+1) !} a_{j-k} \\
& =a_{j+1} \cdot \log ^{j+1}(n) .
\end{aligned}
$$

This shows (A.1) for $j+1$. Finally, we compute using (A.1) for $j+1$ :

$$
\begin{aligned}
\int_{i_{0}}^{n} \frac{1}{i_{1}} & \int_{i_{1}}^{n} \frac{1}{i_{2}} \cdot \ldots \cdot \int_{i_{j}}^{n} \frac{1}{i_{j+1}} \mathrm{~d} i_{j+1} \cdot \ldots \cdot \mathrm{d} i_{1} \\
& =a_{j+1} \cdot \log ^{j+1}(n)-\sum_{k=0}^{j} \frac{(-1)^{k}}{(k+1) !} a_{j-k} \log ^{j-k}(n) \log ^{k+1}\left(i_{0}\right) \\
& =a_{j+1} \cdot \log ^{j+1}(n)+\sum_{k=0}^{j} \frac{(-1)^{k+1}}{(k+1) !} a_{j-k} \log ^{j-k}(n) \log ^{k+1}\left(i_{0}\right) \\
& =a_{j+1} \cdot \log ^{j+1}(n)+\sum_{k=1}^{j+1} \frac{(-1)^{k}}{(k) !} a_{j+1-k} \log ^{j+1-k}(n) \log ^{k}\left(i_{0}\right) \\
& =\sum_{k=0}^{j+1} \frac{(-1)^{k}}{(k) !} a_{j+1-k} \log ^{j+1-k}(n) \log ^{k}\left(i_{0}\right),
\end{aligned}
$$

thus showing (A.2) for $j+1$. This completes the proof of Step 1 .

Step 2. We prove that $a_{j}=\frac{1}{j !}$ for every $j \in \mathbb{N}$ by induction over $j \in \mathbb{N}$. Thus, assume that the claim be true up to $j$ and compute using the recursive relation:

$$
\begin{aligned}
a_{j+1} & =\sum_{k=0}^{j} \frac{(-1)^{k}}{(k+1) !} a_{j-k} \\
& =\sum_{k=0}^{j} \frac{(-1)^{k}}{(k+1) !} \frac{1}{(j-k) !}
\end{aligned}
$$




$$
\begin{aligned}
& =\sum_{k=0}^{j} \frac{(-1)^{k}}{(j+1) !}\left(\begin{array}{c}
j+1 \\
k+1
\end{array}\right) \\
& =\frac{1}{(j+1) !} \sum_{k=0}^{j}(-1)^{k}\left(\begin{array}{c}
j+1 \\
k+1
\end{array}\right) \\
& =\frac{1}{(j+1) !} \sum_{k=1}^{j+1}(-1)^{k-1}\left(\begin{array}{c}
j+1 \\
k
\end{array}\right) \\
& =\frac{1}{(j+1) !}\left(1+\sum_{k=0}^{j+1}(-1)^{k-1}\left(\begin{array}{c}
j+1 \\
k
\end{array}\right)\right) \\
& =\frac{1}{(j+1) !}
\end{aligned}
$$

where the last equality follows from the binomial theorem.

\section{References}

1. Albouy, A., Chenciner, A.: Le probléme des n corps et les distances mutuelles. (French) [The n-body problem and mutual distances]. Invent. Math. 131(1), 151-184 (1998)

2. Albouy, A., Dullin, H.R.: Relative equilibria of the 3-body problem in $\mathbb{R}^{4}$. J. Geom. Mech. 12(3), 323-341 (2020)

3. Asselle, L., Fenucci, M., Portaluri, A.: Bifurcation of balanced configurations for the Newtonian $n$ body problem in $\mathbb{R}^{4}(2020)$, to appear in JFPTA, Viterbo Festschrift, 2022. Preprint available at arXiv:2011.09291

4. Cohen, Frederick R.: Introduction to configuration spaces and their applications. Braids, 183-261, Lect. Notes Ser. Inst. Math. Sci. Natl. Univ. Singap., 19, World Sci. Publ., Hackensack (2010)

5. Fadell, E., Husseini, S.: Geometry and Topology of Configuration Spaces. Springer Monographs in Mathematics. Springer-Verlag, Berlin (2001)

6. Fadell, E., Neuwirth, L.: Configuration spaces. Math. Scand. 10, 111-118 (1962)

7. McCord, C.: Planar central configuration estimates in the $N$-body problem Ergodic Theory Dyn. Systems 16, 1059-1070 (1996)

8. Merkel, J.: Conrad Morse theory and central configurations in the spatial $N$-body problem. J. Dyn. Diff. Equat. 20, 653-668 (2008)

9. Moeckel, R.: Central Configurations. Lecture notes given at the Centre de Recerca Matemãtica (CRM), Barcelona (2014)

10. Moeckel, R.: Lectures on Celestial Mechanics (especially on Central configurations). Preprint available at http://www-users.math.umn.edu/ rmoeckel/notes/Notes.html

11. Pacella, F.: Equivariant Morse theory for flows and an application to the $N$-body problem. Trans. Am. Math. Soc. 297(1), 41-52 (1986)

Publisher's Note Springer Nature remains neutral with regard to jurisdictional claims in published maps and institutional affiliations. 